\title{
The Eocene Hieroglyphic beds and Green shales in the Rożnów Lake area (Silesian Nappe, Outer Carpathians) - facies development and biostratigraphy
}

\author{
Anna Waśkowska \\ AGH University of Science and Technology, Faculty of Geology, Geophysics and Environmental Protection; \\ al Mickiewicza 30, 30-059 Krakow; e-mail: mwaskowsk@agh.edu.pl
}

(C) 2014 Authors. This is an open access publication, which can be used, distributed and reproduced in any medium according to the Creative Commons CC-BY 4.0 License requiring that the original work has been properly cited.

Received: 19 November 2013; accepted: 11 February 2014

\begin{abstract}
In the lithostratigraphic section of a sedimentary sequence of the Silesian Nappe in the area of Rożnów Lake, the strata that occur above the Ciężkowice sandstone and below the Globigerina marls are represented by a relatively thin unit informally named the Hieroglyphic beds and a succeeding thick complex of so-called Green shales. The Hieroglyphic beds, deposited over a short time interval during the Lutetian time (Reticulophragmium amplectens zone), are followed by the Green shales that were deposited from the Lutetian to the Priabonian (Reticulophrgamium amplectens, Ammodisus latus, Reticulophragmium rotundidorstum zones). This work describes the foraminiferal assemblages, on the basis of which, the age of the Green shales was established that in the area of Rożnów Lake. Deposition of the Green shale unit started in the Lutetian, while in other regions of the Silesian Basin it is documented from the Priabonian. The upper boundary of the Green shales is isochronous and defined by the base of the calcareous horizon of a specific type of marls known as the Globigerina marls.
\end{abstract}

Keywords: Hieroglyphic beds, Green shales, Silesian Nappe, Eocene, foraminifera, biostratigraphy

\section{INTRODUCTION}

In the Eocene, deposition of strata typified by the Hieroglyphic beds represented by thin-bedded, sandy-to-shaly turbidites was common within the basins of the Outer Carpathians. This resulted in complexes of mudstones associated with thin sandstone beds, which are defined as the Beloveza beds (e.g. Węcławik 1969, Bogacz et al. 1979, Golonka \& Waśkowska 2012, and citation therein) in the Magura Nappe and as the Hieroglyphic beds in the Silesian, Dukla, Skole, and Subsilesian nappes (e.g. Geroch 1960, Bieda et al. 1973, Morgiel \& Szymakowska 1978, Geroch \& Koszarski 1988, Ryłko 2004 and citation therein). Up section they grade into homogeneous mudstone complexes called the Green shales. The Hieroglyphic beds and the Green shales are informal subdivisions traditionally functioning in the lithostratigraphic nomenclature of the sedimentary successions of the Outer Carpathians. In the Silesian Nappe, these units are defined by the Eocene-age deposits that occur above the Ciężkowice sandstone and below the Globigerina marls (e.g. Guzik \& Pożaryski 1949, Ślączka 1959, Geroch 1960, Bieda et al. 1963, Burtan \& Skoczylas-Ciszewska 1964, Koszarski 1966, Koszarski \& Żytko 1966, Geroch et al. 1967, Golonka \& Waśkowska-Oliwa 2007, Golonka et al. 2013). The distinguishing feature of the Hieroglyphic beds in the Silesian Nappe is 
their development as a thin-bedded flysch facies, in which predominant mudstones are intercalated with thin sandstone beds. Numerous organic hieroglyphs are the typical feature of the sandstone beds, which is emphasised by the name of the unit (Paul \& Tietze 1879).

Lithostratigraphic sections from various regions of the Silesian Nappe document variations in stratigraphy and lithological development of these units. In some regions of the Carpathians, the chief characteristics of the Hieroglyphic beds, that is the thin-bedded flysch facies, is partly replaced by a sequence of green mudstones in which sandstone intercalations are either subordinate or completely absent. The region of Rożnów Lake is one example of such lithological variation, and the succession outcropping in that area is the subject of the present study.

\section{STUDY AREA}

The presented research was conducted on the eastern side of Rożnów Lake, in the area geographically belonging to the Rożnów Foothills. The discussed area is a part of the region underlain by sedimentary sequences of the Silesian Nappe (Fig. 1) - one of the major tectonic units of the Outer Carpathians. The Silesian Nappe is represented in the studied area by a sedimentary succession (the Silesian Series), the profile of which spans the age interval from the Valanginian to the Oligocene, and records the geological history of the Silesian Basin in this period (Burtan \& Skoczylas-Ciszewska 1964, Burtan et al. 1981, Cieszkowski et al. 1981, Leszczyński \& Radomski 1984, Cieszkowski 1992). The Hieroglyphic beds and the Green shales were examined in the belt of outcrops between the Łososina River and the town of Ciężkowice. Samples were collected in the western part of this belt, from natural exposures located along the eastern shores of Rożnów Lake in the village of Sienna, and in stream valleys in the village of Lipie. A section of the stream Szczecinówka flowing down the Przydonicki Watershed (localities Lipie - Gródek) was subjected to detailed analysis (Fig. 1).

The examination area is underlain by the asymmetrical Rożnów anticline (Sokołowski
1935, Książkiewicz 1972, Cieszkowski 1992), called also the Rożnów fold. In the southern limb of this structure, where layers are dipping quite gently $10-30^{\circ}$ towards the south, a complete sequence of strata of the Senonian-Oligocene age is outcropping. The Hieroglyphic beds and Green shales, associated by the underlying and overlying strata, reach the land surface between two dislocations that partly deform the southern limb of the Rożnów fold. These dislocations are: GórowaCiężkowice thrust in the north and Przydonica thrust in the south. Both thrusts form tectonic dislocations of small amplitude (Cieszkowski 1992) (Fig. 1). The belt of the Hieroglyphic beds and Green shales occurs in the synclinal zone and is offset by numerous transverse faults (Cieszkowski 1992). Detailed fieldwork has shown that in the centre of this belt, the axial part of which contains the Globigerina marls and a basal part of the Menilite beds, there is another, small tectonic dislocation related probably to a deformation in the central part of an inconspicuous local syncline (Fig. 1).

\section{MATERIAL AND METHODS}

For the petrographic and micropalaeontological investigations, 23 samples of muddy and clayey sediments were taken. A section of the Green shales was sampled together with immediately underlying and overlying strata. The analysed samples, weighing $0.5 \mathrm{~kg}$ each were treated according to the standard micropalaeontological procedure: they were macerated in an aqueous solution of Glauber's salt, and then washed on $0.63 \mathrm{~mm}$ mesh size sieves. All foraminiferal tests were hand-separated from the obtained residue and underwent taxonomical verification. The foraminiferal assemblages are well preserved; the numbers of tests are high, ranging from 900 to 4,500 specimens per sample of Green shales and 250-370 specimens per sample of the Hieroglyphic beds. Out of a part of the sampled material, polished slabs and 10 thin sections were made for petrographic analyses. The carbonate content was analysed with the volumetric Scheibler method. The TOC content and kerogen provenance by using Rock Eval pyrolysis were analysed on five samples. 

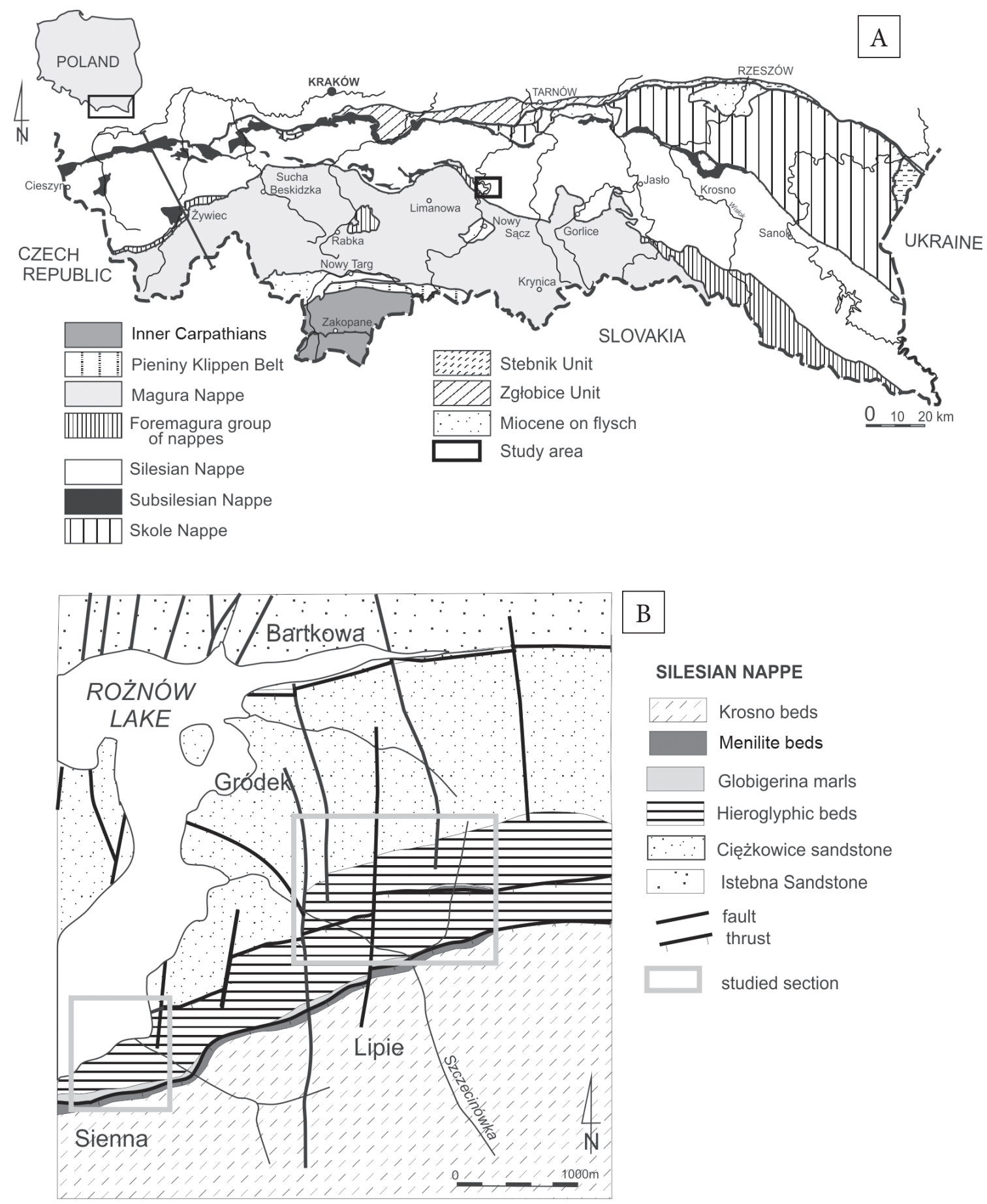

Fig. 1. Location of the studied section on the tectonic sketch-map of Polish Carpathians (A) and on the geological map of the Rożnów Lake area (map on the base of Cieszkowski 1992) (B)

\section{LITHOLOGY}

In the Lipie profile and in the bank sections of Rożnów Lake in the town of Gródek, the sequence between the Ciężkowice sandstone and the Globigerina marls and Menilite beds is dominated by mudstones (Fig. 2).

\section{The Hieroglyphic beds}

Above the Ciężkowice sandstone, which in the examined area forms two thickly-bedded complexes of sandstones interbeded with complexes of variegated shales and packages of sandstones and shales, there is a sequence of thin- and mediumbedded flysch strata (Cieszkowski 1992) (Figs 2, 3). 

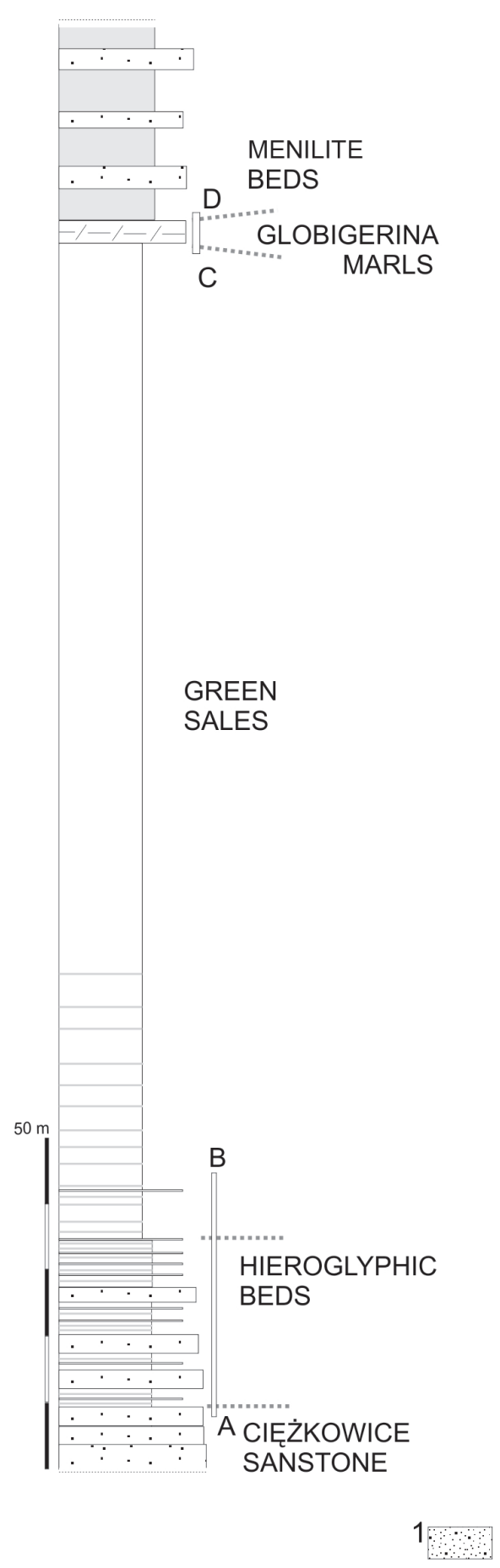

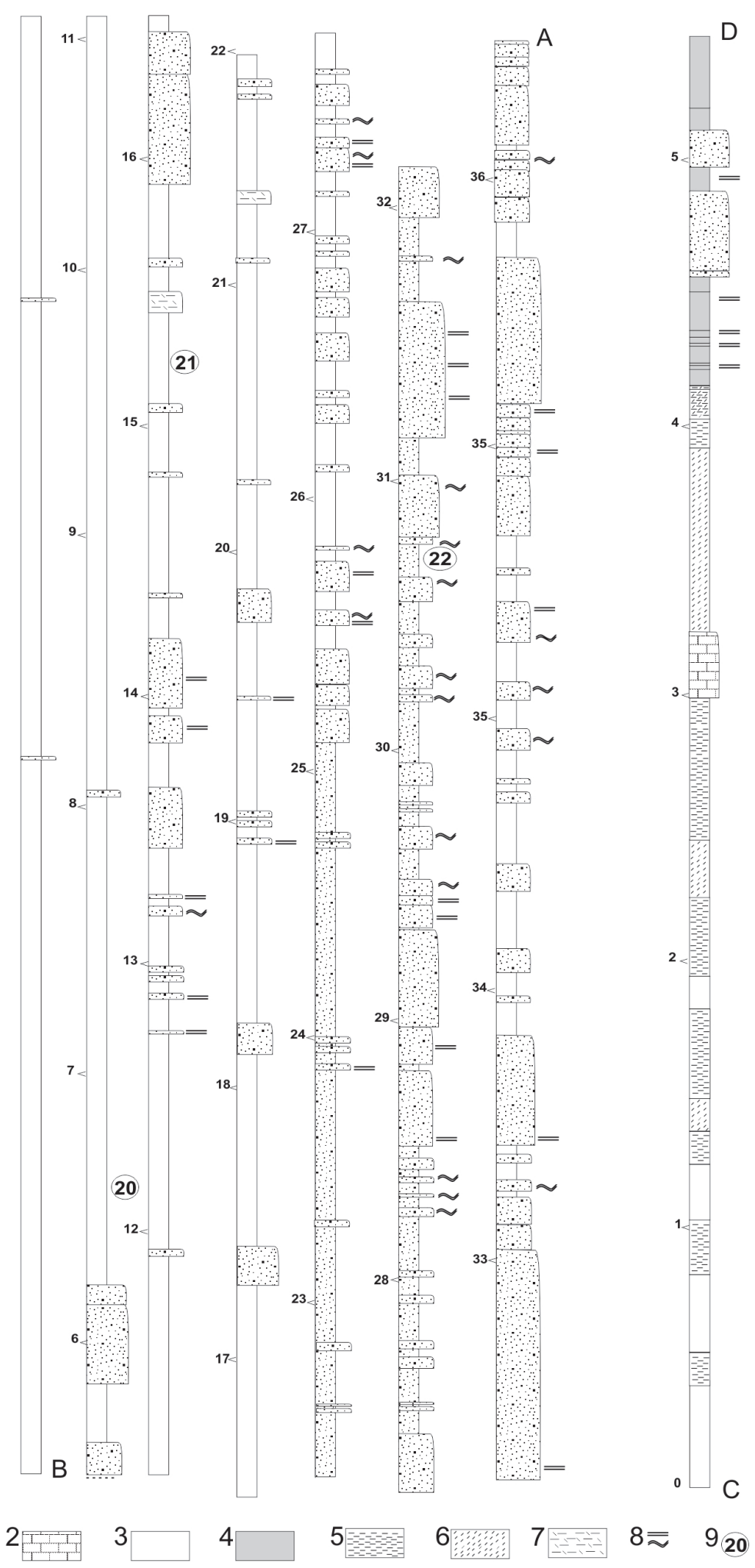

Fig. 2. Profile of Middle Eocene-Oligocene deposits in the Rożnów Lake area (Szczecinówka valley), with detailed logs of the Hieroglyphic beds $(A-B)$ and the topmost part of Green shales and Globigerina marls (C-D): 1 - sandstone, 2 - hard marl, 3 - green-gray shale, 4 - brown shale, 5 - soft marl, 6 - green marly shale, 7 -siderite, 8 -parallel lamination, 9 -foraminiferal samples 

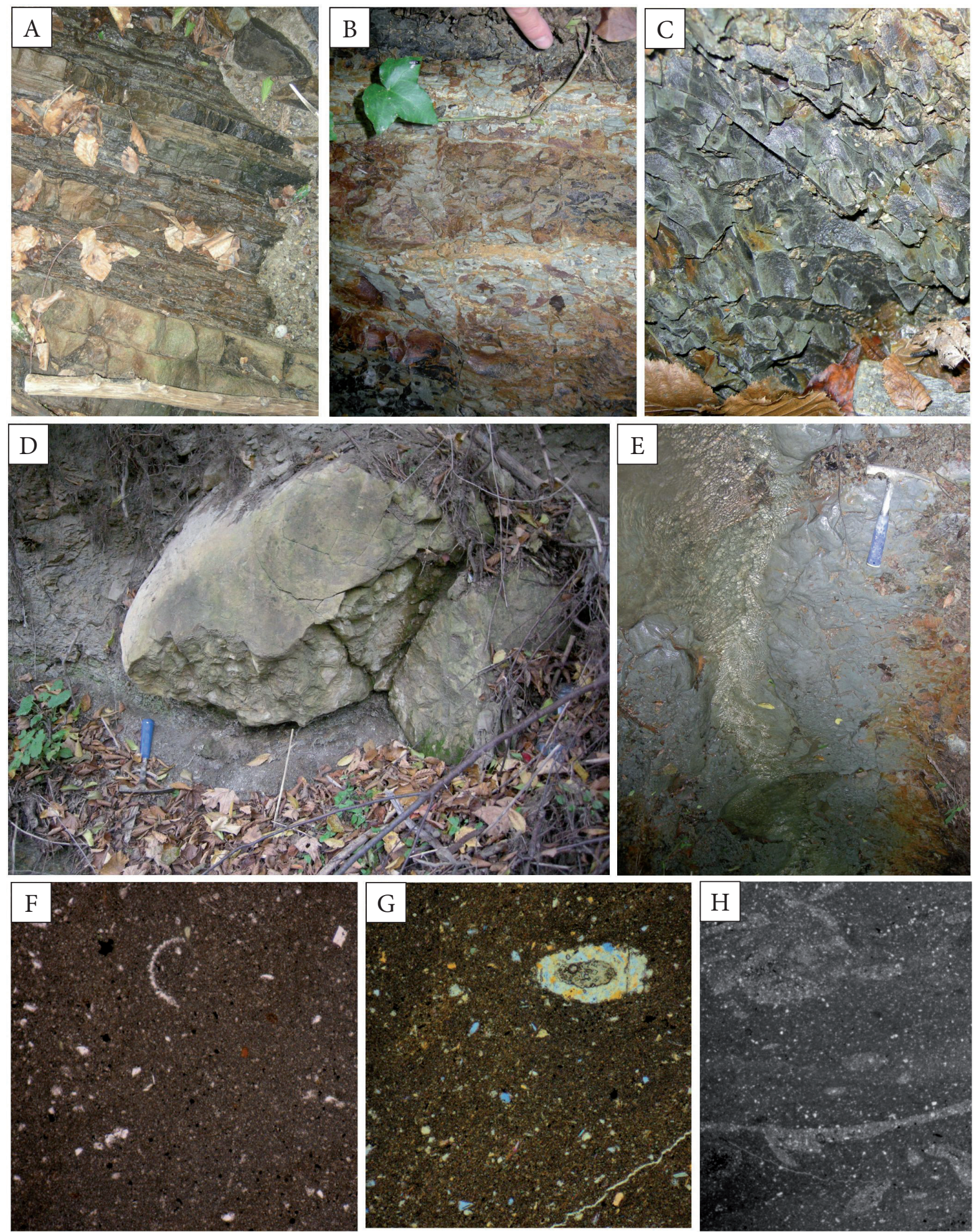

Fig. 3. A) thin-bedded flysch of the Hieroglyphic beds; B) gray and brown mudstones in the lower part of the Green shales; C) green and grey shales in the middle part of the Green shales; D) calcareous bowl in the middle part of the Green shales; E) green and grey shales in the upper part of the Green shales; thin sections: F) mudstones with foraminiferal test fragments (upper part of the Green shales); G) mudstones with Bathysiphon sp. (middle part of the Green shales); E) bioturbations within mudstones (middle part of the Green shales) 
In Rożnów Lake, the topmost part of the Ciężkowice sandstone consists of complexes of thick-, medium- and thin-bedded grey quartz arenites, intercalated with intervals of thin-bedded flysch facies. The stratigraphic transition to the overlying mudstone is represented by a fining-upwards, $20 \mathrm{~m}$ thick complex of facies of the Hieroglyphic beds type, with a gradually decreasing number and thickness of sandstone intercalations, and an increasing proportion of mudstone. The mudstones are laminated, locally sandy and with muscovite flakes seen on cleavage surfaces, non-calcareous, dark grey in colour, interlaminated with dark mudstones and with cleavage surfaces covered by a rusty limonitic coating. The sandstones are grey in colour; a medium- to thin-bedded variety predominates in the lower part and a thin- to very thinly-bedded type in the upper part. However, medium thick sandstone beds appear irregularly throughout the section. Such beds also occur in the top part of the profile where they define the end of the sedimentation of the thin-bedded flysch facies.

The sandstone beds are intercalated with mudstones and sporadically occur as clusters of two amalgamated sandstone beds. The sandstones represent quartzarenites, are fine grained, with parallel and wavy lamination and organic hieroglyphs commonly occurring on bed soles. Sideritic concretions, pelitic, elongated, a few centimetres thick occur occasionally within the mudstones. The thickness of the mudstone complexes increases up the section.

\section{The Green shales}

The Green shales occur above the Hieroglyphic beds (Figs 2, 3). Their lower part is represented by laminated massive muddy shales and grey to grey-green massive muddy shales, occasionally intercalated with laminated dark grey shales. Upwards within the section, the dark-coloured shales disappear; mudstones become softer and locally pass into claystones. Non-calcareous grey and grey-green mudstones and claystones prevail in the section; slightly marly varieties occur only occasionally. Sporadically, very thin bentonite layers cream in colour, appear between the mudstones. The total thickness of the Green shales complex is estimated to be ca. $150 \mathrm{~m}$.
Relatively large oval limestone clasts ranging from over $10 \mathrm{~cm}$ to $1.5 \mathrm{~m}$ in diameter are an integral component of the section (Fig. 3). They have smooth surfaces, oval shapes, are cut by thick veins filled with crystalline calcite. The limestones are grey in colour but weather light grey; macroscopically they are composed of pelite and fine crystalline sparite. Two kinds of limestone were identified: one recrystallized, with sparite texture, and a second - marly, micritic with bioclasts of shells of the Palaeogene-age planktonic foraminifera. The mudstones adjacent to the limestone clasts are plastically deformed. A closer inspection of the clasts suggests that they resulted from submarine landslides, with fragments of broken-up limestone beds rolling over sea bottom, thus attaining the oval shapes.

Grey-green and green marly (the carbonate content in the top part increases from a few up to $300 \mathrm{~g} / \mathrm{kg}$ ) mudstones appear in the highest part of the profile, between mudstones (Fig. 4).

The TOC value in the Green shales amounts to $0.2-0.5 \%$. At Sienna, in lakeside outcrops of Rożnów Lake, an olistostrome of considerable size was documented. It contains, apart from clasts of crystalline rocks and limestones, a significant proportion of Early Eocene mudstones, banded sandstones with calcareous fauna, and olistoliths of flysch packages and of banded sandstones with calcareous fauna (Cieszkowski \& Waśkowska 2013a, b).

The thin section analysis of the Green shales shows that they are homogeneous, with clay minerals predominant and a variable content of terrigenous quartz grains (Fig. 3). The grain-size and content of the quartz grains decrease up the succession. This is reflected by the proportion of the insoluble residue of grains exceeding $0.63 \mathrm{~mm}$ in diameter decreasing upwards the analysed section (Fig. 4). Fine glauconite aggregates are an accessory component. The biogenic constituent is subordinate and represented by fragments of planktonic foraminifera tests present in the upper part of the succession. Apart from common occurrences of Bathysiphon or Nothia usually preserved in situ, agglutinated foraminifera are observed in thin sections very rarely (Fig. 3). However, taxonomically variable assemblages of agglutinated foraminifera were obtained from the insoluble 
residue of the analysed mudstones (Tab. 1). They were accompanied by accessory fish teeth; solitary spines of echinoids (sea urchins) were observed within pellets. Delicate lamination in the lower part of the shale succession is affected by bioturbation but above, bioturbation is extremely common and the lamination disappears (Fig. 3). The bioturbations are filled with sediment of coarser fraction, in which quartz grains prevail. Narrow zones surrounding the bioturbations are enriched with concentrations of fine aggregates and fine crystals of autigenic pyrite.
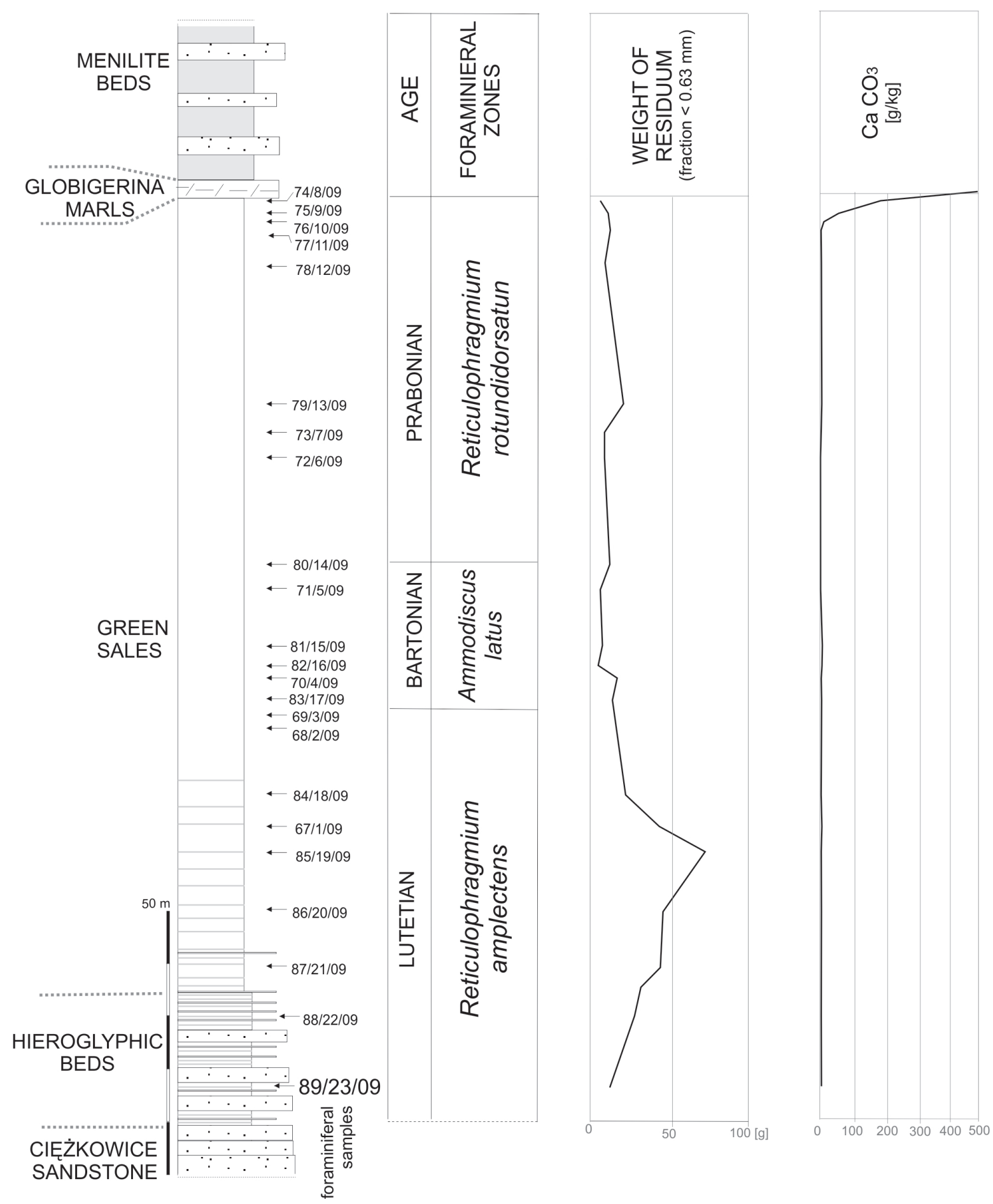

Fig. 4. Profile of Middle-Upper Eocene deposits in the Rożnów Lake area with distribution of biostratigraphical zones, CaCa content, and amount of clastic material 
Table 1

Taxonomical composition of foraminiferal samples (samples position on Figure 4). I: 1-4 specimens per sample; II: 5-9; V: 10-19; X: 20-49; W: 50-99; Z: 100-199; H: 200-499; F: 500-999; M: >1000

\begin{tabular}{|c|c|c|c|c|c|c|c|c|c|c|c|c|c|c|c|c|c|c|c|c|c|c|c|}
\hline \multirow[b]{2}{*}{ Species } & \multicolumn{23}{|c|}{ Sample number } \\
\hline & 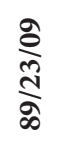 & 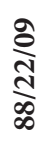 & 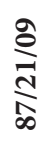 & 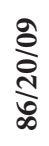 & $\underset{\infty}{\frac{2}{2}}$ & $\stackrel{8}{\frac{2}{6}}$ & $\stackrel{\substack{\infty \\
+\infty}}{+\infty}$ & 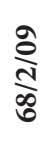 & $\frac{\frac{\partial}{m}}{\frac{m}{2}}$ & 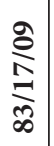 & $\underset{\substack{\infty \\
\infty}}{\stackrel{2}{6}}$ & $\frac{2}{\frac{2}{R}}$ & $\sum_{\infty}^{\infty}$ & $\frac{2}{n}$ & 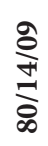 & 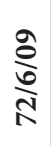 & $\frac{a}{\frac{2}{n}}$ & $\frac{\stackrel{a}{m}}{\frac{2}{2}}$ & $\underset{\substack{\infty \\
\stackrel{2}{a}}}{\stackrel{2}{a}}$ & 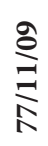 & $\frac{\substack{0 \\
0}}{\circ}$ & $\frac{a}{2}$ & 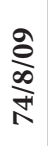 \\
\hline 1 & 2 & 3 & 4 & 5 & 6 & 7 & 8 & 9 & 10 & 11 & 12 & 13 & 14 & 15 & 16 & 17 & 18 & 19 & 20 & 21 & 22 & 23 & 24 \\
\hline $\begin{array}{l}\text { Adercotryma } \\
\text { agterbergi Gradstein } \\
\text { et Kaminski }\end{array}$ & & II & & & & & & & & & & & & & & & & & & & & & \\
\hline $\begin{array}{l}\text { Ammobaculites } \\
\text { agglutinans } \\
\text { d'Orbignyi }\end{array}$ & & & & & & & & & & & & & & $\mathrm{V}$ & & & I & & & & & & \\
\hline $\begin{array}{l}\text { Ammodiscus } \\
\text { cretaceus (Reuss) }\end{array}$ & & & I & & I & & & I & $\mathrm{I}$ & I & $\mathrm{V}$ & & $\mathrm{X}$ & $\mathrm{V}$ & W & $\mathrm{V}$ & I & & II & W & I & $\mathrm{X}$ & $\mathrm{W}$ \\
\hline $\begin{array}{l}\text { Ammodiscus latus } \\
\text { Grzybowski }\end{array}$ & & & & & I & & & & & II & $\mathrm{X}$ & & W & I & W & $\mathrm{V}$ & I & $\mathrm{W}$ & V & W & $\mathrm{V}$ & $\mathrm{X}$ & \\
\hline $\begin{array}{l}\text { Ammodiscus } \\
\text { peruvianus Berry }\end{array}$ & & & & & $\mathrm{V}$ & & $\mathrm{V}$ & II & $\mathrm{X}$ & $\mathrm{V}$ & $\mathrm{V}$ & II & $\mathrm{V}$ & II & $\mathrm{X}$ & $\mathrm{V}$ & II & $\mathrm{H}$ & II & W & $\mathrm{V}$ & $\mathrm{X}$ & $\mathrm{X}$ \\
\hline $\begin{array}{l}\text { Ammodiscus } \\
\text { tenuissimus } \\
\text { Grzybowski }\end{array}$ & & & I & & I & & & I & & $\mathrm{V}$ & & & II & & $\mathrm{V}$ & $\mathrm{W}$ & I & & & W & $\mathrm{V}$ & & \\
\hline Ammodiscus sp. & & & & & & II & & & & & II & & & II & II & $\mathrm{V}$ & I & II & & & I & $\mathrm{W}$ & I \\
\hline $\begin{array}{l}\text { Ammolagena clavata } \\
\text { (Jones et Parker) }\end{array}$ & I & & & I & & & II & & & & II & & $\mathrm{I}$ & II & II & & & $\mathrm{X}$ & $\mathrm{I}$ & $\mathrm{I}$ & & & \\
\hline $\begin{array}{l}\text { Ammomarginulina } \\
\text { aubertae Gradstein et } \\
\text { Kaminski }\end{array}$ & & & & & & & & & & & & & & II & & & & & & & & & \\
\hline $\begin{array}{l}\text { Ammosphaeroidina } \\
\text { pseudopauciloculata } \\
\text { (Mjatliuk) }\end{array}$ & & $\mathrm{X}$ & $\mathrm{X}$ & $\mathrm{X}$ & M & & W & W & $\mathrm{W}$ & W & $\mathrm{X}$ & W & $\mathrm{H}$ & II & $\mathrm{F}$ & $\mathrm{V}$ & W & & & & $\mathrm{V}$ & & \\
\hline $\begin{array}{l}\text { Annectina biedai } \\
\text { Gradstein et } \\
\text { Kaminski }\end{array}$ & & & & & & & & & I & & $\mathrm{I}$ & & & I & & & & & & & & I & \\
\hline $\begin{array}{l}\text { Arenobulimmina } \\
\text { dorbignyi (Reuss) }\end{array}$ & & & & & & & & & & & & & & & & & & & & & & & $\mathrm{W}$ \\
\hline $\begin{array}{l}\text { Bathysiphon sp. and } \\
\text { Nothia div. sp. }\end{array}$ & VV & II & $\mathrm{Z}$ & $\mathrm{H}$ & $\mathrm{W}$ & $\mathrm{F}$ & $\mathrm{Z}$ & $\mathrm{Z}$ & $\mathrm{W}$ & $\mathrm{H}$ & $\mathrm{H}$ & $\mathrm{H}$ & $\mathrm{H}$ & $\mathrm{F}$ & $\mathrm{H}$ & $\mathrm{F}$ & $\mathrm{H}$ & $\mathrm{M}$ & $\mathrm{F}$ & $\mathrm{F}$ & $\mathrm{W}$ & $\mathrm{W}$ & $\mathrm{H}$ \\
\hline Bulbobaculites sp 1. & & & & $\mathrm{I}$ & & & & & & & & & & & I & & I & II & $\mathrm{I}$ & I & & & \\
\hline $\begin{array}{l}\text { Buzasina pacifica } \\
\text { (Krasheninnikov) }\end{array}$ & & & I & $\mathrm{V}$ & & & & & & & & & $\mathrm{I}$ & & & & & & & & & & II \\
\hline $\begin{array}{l}\text { Caudammina ovula } \\
\text { (Grzybowski) }\end{array}$ & & & & & I & & & & & & & & & & & & & & & & & & \\
\hline Caudammina sp. & & I & & & & & & & & & & & & & & & & & & & & & \\
\hline
\end{tabular}


Table 1 cont.

\begin{tabular}{|c|c|c|c|c|c|c|c|c|c|c|c|c|c|c|c|c|c|c|c|c|c|c|c|}
\hline 1 & 2 & 3 & 4 & 5 & 6 & 7 & 8 & 9 & 10 & 11 & 12 & 13 & 14 & 15 & 16 & 17 & 18 & 19 & 20 & 21 & 22 & 23 & 24 \\
\hline Cibicides/Eponides spp. & & & & & & & & & & & & & & & & & & & & & & & $\mathrm{V}$ \\
\hline $\begin{array}{l}\text { Cribrostomoides } \\
\text { subglobosus } \\
\text { (Cushaman) }\end{array}$ & $\mathrm{X}$ & & $\mathrm{X}$ & $\mathrm{X}$ & & & W & $\mathrm{X}$ & I & & & $\mathrm{V}$ & I & $\mathrm{V}$ & I & $\mathrm{W}$ & $X$ & $\mathrm{X}$ & II & $\mathrm{V}$ & $\mathrm{W}$ & $\mathrm{W}$ & $\mathrm{F}$ \\
\hline $\begin{array}{l}\text { Cyclammina placenta } \\
\text { (Reuss) }\end{array}$ & & & & & & & & & & & & & & & I & & $\mathrm{I}$ & & & & & & \\
\hline $\begin{array}{l}\text { Cyclamminal } \\
\text { Reticulophragmium sp. }\end{array}$ & & & & & & & & & & & & & & I & & & I & & & & & & I \\
\hline Cystammina sp. & & I & & & & & $\mathrm{X}$ & & & & & & & $\mathrm{Z}$ & I & & & & & $\mathrm{V}$ & & & \\
\hline Dolgenia sp. & I & I & $\mathrm{X}$ & & I & $\mathrm{V}$ & $\mathrm{V}$ & II & I & $\mathrm{X}$ & $\mathrm{V}$ & $\mathrm{X}$ & I & $\mathrm{Z}$ & $\mathrm{W}$ & $\mathrm{V}$ & $\mathrm{V}$ & $\mathrm{V}$ & $\mathrm{I}$ & W & & I & \\
\hline $\begin{array}{l}\text { Eratidus gerochi } \\
\text { Kaminski et } \\
\text { Gradstein }\end{array}$ & & & & & & & & & & & & & & & & & I & & & & & & \\
\hline Eggerelloides sp. & & & & & & & & & $\mathrm{X}$ & & & II & & I & & & & & & & & & \\
\hline $\begin{array}{l}\text { Glomospira charoides } \\
\text { (Jones et Parker) }\end{array}$ & I & X & X & $\mathrm{X}$ & I & $\mathrm{X}$ & $\mathrm{V}$ & $\mathrm{I}$ & $\mathrm{X}$ & $\mathrm{X}$ & W & $\mathrm{X}$ & $\mathrm{X}$ & $\mathrm{H}$ & $\mathrm{W}$ & $X$ & $\mathrm{~W}$ & $\mathrm{X}$ & $\mathrm{V}$ & $\mathrm{V}$ & $\mathrm{V}$ & $\mathrm{W}$ & $\mathrm{H}$ \\
\hline $\begin{array}{l}\text { Glomospira glomerata } \\
\text { (Grzybowski) }\end{array}$ & & II & & & $\mathrm{V}$ & & & $\mathrm{V}$ & $\mathrm{X}$ & $\mathrm{W}$ & $\mathrm{X}$ & $\mathrm{X}$ & $\mathrm{X}$ & $\mathrm{W}$ & $\mathrm{W}$ & $\mathrm{V}$ & $\mathrm{X}$ & & & & & II & $\mathrm{W}$ \\
\hline $\begin{array}{l}\text { Glomospira gordialis } \\
\text { (Jones et Parker) }\end{array}$ & II & I & $\mathrm{X}$ & II & V & $\mathrm{X}$ & & & $\mathrm{V}$ & & $\mathrm{X}$ & & $\mathrm{X}$ & I & $\mathrm{X}$ & $\mathrm{X}$ & $\mathrm{V}$ & W & I & $\mathrm{X}$ & $\mathrm{V}$ & $\mathrm{X}$ & $\mathrm{X}$ \\
\hline $\begin{array}{l}\text { Glomospira serpens } \\
\text { (Grzybowski) }\end{array}$ & & & & & & & & & II & & I & & I & I & V & & II & & & $\mathrm{I}$ & & I & $\mathrm{I}$ \\
\hline Glomospira sp. & & & & & & & & & & & & & & I & & & & I & & $\mathrm{V}$ & & & \\
\hline $\begin{array}{l}\text { Gyroidinoides } \\
\text { globosus (Hagenow) }\end{array}$ & & & & & & & & & & & & & & & & & & & & & & & $\mathrm{I}$ \\
\hline $\begin{array}{l}\text { Haplophragmoides } \\
\text { excavatus Cushman }\end{array}$ & & & V & & & & $\mathrm{X}$ & & & & & & & & & & & & & & & & \\
\hline $\begin{array}{l}\text { Haplophragmoides } \\
\text { horridus } \\
\text { (Grzybowski) }\end{array}$ & II & & & V & & & & $\mathrm{V}$ & & & & & & & & & I & & & & & & \\
\hline $\begin{array}{l}\text { Haplophragmoides } \\
\text { kirki Wickenden }\end{array}$ & & & & & & & II & & & $\mathrm{V}$ & & & II & & & $\mathrm{V}$ & W & & & V & W & $\mathrm{X}$ & II \\
\hline $\begin{array}{l}\text { Haplophragmoides } \\
\text { nauticus Kender, } \\
\text { Kaminski et Jones }\end{array}$ & I & I & I & $\mathrm{X}$ & VII & $\mathrm{W}$ & $\mathrm{X}$ & & II & & II & II & $\mathrm{W}$ & & & & & & & & & X & \\
\hline $\begin{array}{l}\text { Haplophragmoides } \\
\text { parvulus Blaicher }\end{array}$ & & & X & & $\mathrm{X}$ & & & I & & I & & & & II & & $\mathrm{V}$ & & I & & & & & \\
\hline $\begin{array}{l}\text { Haplophragmoides } \\
\text { porrectus Maslakova }\end{array}$ & & & & & & & & & & & & & & & & & $\mathrm{X}$ & & & & I & & \\
\hline $\begin{array}{l}\text { Haplophragmoides } \\
\text { stomatus } \\
\text { (Grzybowski) }\end{array}$ & & & & $\mathrm{V}$ & & & & & & & & & & & II & & II & & $\mathrm{X}$ & & $\mathrm{X}$ & & V \\
\hline
\end{tabular}


Table 1 cont.

\begin{tabular}{|c|c|c|c|c|c|c|c|c|c|c|c|c|c|c|c|c|c|c|c|c|c|c|c|}
\hline \multirow[b]{2}{*}{ Species } & \multicolumn{23}{|c|}{ Sample number } \\
\hline & 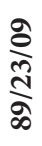 & $\underset{\substack{\text { సे } \\
\infty}}{\stackrel{\infty}{\infty}}$ & 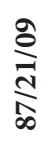 & $\underset{\substack{\text { d } \\
\infty}}{\infty}$ & $\frac{2}{2}$ & 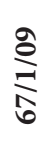 & $\underset{\infty}{\stackrel{\infty}{\infty}}$ & 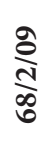 & $\frac{2}{\frac{m}{g}}$ & $\frac{a}{\infty}$ & $\frac{\substack{0 \\
\frac{2}{c}}}{\infty}$ & 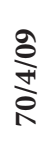 & $\sum_{\infty}^{\frac{9}{10}}$ & $\frac{2}{\sqrt{n}}$ & 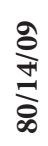 & $\frac{\substack{0 \\
\frac{0}{2}}}{2}$ & $\frac{a}{\frac{2}{n}}$ & $\frac{2}{\frac{2}{2}}$ & $\underset{\substack{\infty \\
\stackrel{2}{a}}}{\stackrel{2}{a}}$ & 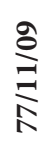 & $\frac{\substack{0 \\
\frac{1}{0}}}{n}$ & $\frac{a}{a}$ & $\frac{\substack{\infty \\
\text { म }}}{n}$ \\
\hline 1 & 2 & 3 & 4 & 5 & 6 & 7 & 8 & 9 & 10 & 11 & 12 & 13 & 14 & 15 & 16 & 17 & 18 & 19 & 20 & 21 & 22 & 23 & 24 \\
\hline $\begin{array}{l}\text { Haplophragmoides } \\
\text { walteri (Grzybowski) }\end{array}$ & $\mathrm{V}$ & I & $\mathrm{V}$ & $\mathrm{V}$ & $\mathrm{V}$ & II & $\mathrm{H}$ & $\mathrm{X}$ & $\mathrm{X}$ & $\mathrm{V}$ & $\mathrm{H}$ & $\mathrm{X}$ & W & $\mathrm{X}$ & $\mathrm{I}$ & $\mathrm{X}$ & $\mathrm{V}$ & & & $\mathrm{I}$ & $\mathrm{I}$ & $\mathrm{H}$ & \\
\hline Haplophragmoides sp. & 1 & $\mathrm{I}$ & & & & & & II & & $\mathrm{X}$ & & & $\mathrm{V}$ & & $\mathrm{X}$ & $\mathrm{X}$ & & & & & & $\mathrm{V}$ & $\mathrm{X}$ \\
\hline $\begin{array}{l}\text { Hormosina } \\
\text { velascoensis } \\
\text { (Cushman) }\end{array}$ & & & & & & & & & I & & & & & & & & & & & & & & \\
\hline $\begin{array}{l}\text { Hormosina } \\
\text { trinitatensis } \\
\text { Cushman et Renz }\end{array}$ & & & & & & & & & & & & & & & & & & & II & & & & \\
\hline $\begin{array}{l}\text { Hormosina } \\
\text { trinitatensis } \\
\text { Cushman et Renz- } \\
\text { single chambers }\end{array}$ & & & & & $\mathrm{V}$ & II & II & & & & $\mathrm{I}$ & I & $\mathrm{I}$ & & & & & I & & & & & II \\
\hline Hormosina sp. & & & & & & & & & & & & & & & & & & II & & & & I & \\
\hline $\begin{array}{l}\text { Hormosinelloides } \\
\text { guttifer (Brady) }\end{array}$ & & & $\mathrm{V}$ & & & & $\mathrm{I}$ & I & & $\mathrm{I}$ & II & & I & II & $\mathrm{I}$ & $\mathrm{X}$ & II & $\mathrm{X}$ & & $\mathrm{X}$ & $\mathrm{I}$ & $\mathrm{I}$ & \\
\hline Hyperammina sp. & & & & & & & I & I & & & & & & & & & II & II & $\mathrm{I}$ & & I & & \\
\hline $\begin{array}{l}\text { Insculptarenula } \\
\text { subvesicularis } \\
\text { (Hanzlikova) }\end{array}$ & & W & & & & $\mathrm{X}$ & & & & & & & & & & & & & & & & & \\
\hline $\begin{array}{l}\text { Karrerulina conversa } \\
\text { (Grzybowski) }\end{array}$ & & & II & & & & $\mathrm{X}$ & & $\mathrm{X}$ & & & $\mathrm{V}$ & & & & $\mathrm{X}$ & II & & & & & & \\
\hline $\begin{array}{l}\text { Karrerulina } \\
\text { coniformis } \\
\text { (Grzybowski) }\end{array}$ & & & & & & $\mathrm{X}$ & $\mathrm{H}$ & $\mathrm{X}$ & $\mathrm{X}$ & V & & & & II & I & $\mathrm{F}$ & II & & & & I & & \\
\hline $\begin{array}{l}\text { Lituotuba lituiformis } \\
\text { (Brady) }\end{array}$ & & I & & & & & & & & & & & & I & & & & & I & II & & & \\
\hline $\begin{array}{l}\text { Ossangularia } \\
\text { plummerae Brotzen }\end{array}$ & & & & & & & & & & & & & I & & & & & & & & & & \\
\hline $\begin{array}{l}\text { Paratrochamminoides } \\
\text { and Trochamminoi- } \\
\text { des div. sp. }\end{array}$ & $\mathrm{X}$ & W & $\mathrm{H}$ & $\mathrm{H}$ & W & $\mathrm{H}$ & $\mathrm{H}$ & $\mathrm{H}$ & $\mathrm{Z}$ & $\mathrm{Z}$ & $\mathrm{M}$ & $\mathrm{H}$ & $\mathrm{H}$ & $\mathrm{M}$ & $F$ & $\mathrm{~F}$ & $\mathrm{H}$ & $\mathrm{F}$ & $\mathrm{X}$ & $\mathrm{F}$ & $\mathrm{V}$ & $\mathrm{Z}$ & $\mathrm{H}$ \\
\hline $\begin{array}{l}\text { Placentammina } \\
\text { placenta } \\
\text { (Grzybowski) }\end{array}$ & I & & $\mathrm{V}$ & $\mathrm{V}$ & I & $\mathrm{V}$ & $\mathrm{V}$ & $\mathrm{X}$ & I & I & & I & II & & II & $\mathrm{X}$ & & II & I & W & W & I & $\mathrm{X}$ \\
\hline $\begin{array}{l}\text { Popovia beckmanni } \\
\text { (Kaminski et Geroch) }\end{array}$ & & I & & I & $\mathrm{X}$ & & $\mathrm{V}$ & & & & I & & II & I & & & & & & & & & \\
\hline $\begin{array}{l}\text { Prasphaerammina } \\
\text { subgaleata Vasicek }\end{array}$ & & & $\mathrm{X}$ & I & & II & $\mathrm{Z}$ & $\mathrm{V}$ & I & I & & & II & $\mathrm{V}$ & II & $\mathrm{X}$ & & & & & & & \\
\hline
\end{tabular}


Table 1 cont.

\begin{tabular}{|c|c|c|c|c|c|c|c|c|c|c|c|c|c|c|c|c|c|c|c|c|c|c|c|}
\hline 1 & 2 & 3 & 4 & 5 & 6 & 7 & 8 & 9 & 10 & 11 & 12 & 13 & 14 & 15 & 16 & 17 & 18 & 19 & 20 & 21 & 22 & 23 & 24 \\
\hline $\begin{array}{l}\text { Psammospiphonella } \\
\text { and Rhabdammina } \\
\text { div. sp (fragments) }\end{array}$ & I & $\mathrm{V}$ & V & $\mathrm{H}$ & W & $\mathrm{X}$ & M & $\mathrm{V}$ & $\mathrm{H}$ & $\mathrm{X}$ & $\mathrm{Z}$ & $\mathrm{X}$ & $\mathrm{H}$ & W & $\mathrm{H}$ & $\mathrm{Z}$ & $\mathrm{X}$ & $\mathrm{H}$ & W & $\mathrm{H}$ & $\mathrm{H}$ & $\mathrm{H}$ & $\mathrm{M}$ \\
\hline Psammosphaera sp. & & I & & I & & & $\mathrm{W}$ & & & I & & II & & I & & & & & & & & & \\
\hline $\begin{array}{l}\text { Pseudonodosinella } \\
\text { elongata } \\
\text { (Grzybowski) }\end{array}$ & & & & & & & $\mathrm{H}$ & & $\mathrm{V}$ & & $X$ & I & $\mathrm{V}$ & I & II & II & I & $\mathrm{X}$ & I & $\mathrm{V}$ & $\mathrm{X}$ & II & $\mathrm{X}$ \\
\hline $\begin{array}{l}\text { Pseudonodosinella } \\
\text { nodulosa (Brady) }\end{array}$ & I & & & & & & & & & & & & I & & & & & & & & & & \\
\hline $\begin{array}{l}\text { Recurvoides div. sp, } \\
\text { Thalmannammina } \\
\text { subturbinata } \\
\text { (Grzybowski), } \\
\text { Recurvoidella lamella } \\
\text { (Grzybowski) }\end{array}$ & $\mathrm{Z}$ & W & $\mathrm{Z}$ & $\mathrm{H}$ & $\mathrm{H}$ & $\mathrm{H}$ & M & $\mathrm{Z}$ & W & $\mathrm{Z}$ & $\mathrm{H}$ & $\mathrm{W}$ & $\mathrm{Z}$ & $\mathrm{H}$ & $\mathrm{H}$ & M & $\mathrm{W}$ & $\mathrm{F}$ & $\mathrm{X}$ & $\mathrm{X}$ & W & $\mathrm{W}$ & Z \\
\hline $\begin{array}{l}\text { Reophax duplex } \\
\text { Grzybowski }\end{array}$ & & & $\mathrm{I}$ & $\mathrm{X}$ & $\mathrm{X}$ & $\mathrm{V}$ & W & $\mathrm{V}$ & I & & & I & $\mathrm{V}$ & I & $\mathrm{X}$ & $\mathrm{V}$ & & $\mathrm{H}$ & $\mathrm{X}$ & $\mathrm{X}$ & $\mathrm{V}$ & $\mathrm{W}$ & $\mathrm{Z}$ \\
\hline $\begin{array}{l}\text { Reophax pilulifer } \\
\text { Brady }\end{array}$ & & & & & I & & $\mathrm{V}$ & & I & & I & & I & I & I & & II & $\mathrm{Z}$ & II & & I & $\mathrm{X}$ & II \\
\hline Reophax sp. & & & & & & & I & & & & & & & I & & & I & & & I & & & \\
\hline $\begin{array}{l}\text { Reophax - single } \\
\text { chambers }\end{array}$ & I & & I & $\mathrm{X}$ & II & $\mathrm{X}$ & $\mathrm{Z}$ & $\mathrm{V}$ & & & $\mathrm{V}$ & II & W & & $\mathrm{Z}$ & $\mathrm{V}$ & $\mathrm{X}$ & $\mathrm{F}$ & $\mathrm{Z}$ & W & $\mathrm{H}$ & $\mathrm{Z}$ & $\mathrm{Z}$ \\
\hline $\begin{array}{l}\text { Reticulophragmium } \\
\text { amplectens } \\
\text { (Grzybowski) }\end{array}$ & & & $X$ & $\mathrm{Z}$ & II & $\mathrm{X}$ & $\mathrm{F}$ & $\mathrm{X}$ & $\mathrm{X}$ & & & $\mathrm{X}$ & & & II & & & & & I & & & \\
\hline $\begin{array}{l}\text { Reticulophragmium } \\
\text { rotindurorsatum } \\
\text { (Hantken) }\end{array}$ & & & & & & & & & & & & & & & I & $\mathrm{V}$ & & $\mathrm{W}$ & & & I & & \\
\hline Reticulophragmium sp. & & & & & I & & & & & & II & & & & II & & & 1 & & & & & \\
\hline $\begin{array}{l}\text { Saccammina } \\
\text { grzybowskii } \\
\text { (Schubert) }\end{array}$ & & & & & & II & & $\mathrm{X}$ & & & & I & & & & & I & $\mathrm{W}$ & & W & & & \\
\hline $\begin{array}{l}\text { Spiroplectammina } \\
\text { spectabilis } \\
\text { (Grzybowski) }\end{array}$ & $\mathrm{X}$ & W & & & & & $\mathrm{H}$ & & & & II & $\mathrm{H}$ & $\mathrm{V}$ & & I & $\mathrm{V}$ & I & & & & & & \\
\hline $\begin{array}{l}\text { Subbotina linaperta } \\
\text { Finlay }\end{array}$ & & & & & & & & & I & & & & & & & & & & & & & & $\mathrm{X}$ \\
\hline $\begin{array}{l}\text { Subreophax scalaris } \\
\text { (Grzybowski) }\end{array}$ & & & I & & $\mathrm{X}$ & II & & I & & & & II & & I & & II & & I & & & & & I \\
\hline $\begin{array}{l}\text { Subreophax } \\
\text { splendidus } \\
\text { (Grzybowski) }\end{array}$ & & & $\mathrm{V}$ & & & & & & & & & $\mathrm{V}$ & & & & $\mathrm{X}$ & I & $\mathrm{X}$ & I & & & & \\
\hline $\begin{array}{l}\text { Subrepohax } \\
\text { pseudoscalaris } \\
\text { (Grzybowski) }\end{array}$ & & & I & & & & & & & & & & & I & I & & & $\mathrm{X}$ & & I & & & \\
\hline
\end{tabular}


Table 1 cont.

\begin{tabular}{|c|c|c|c|c|c|c|c|c|c|c|c|c|c|c|c|c|c|c|c|c|c|c|c|}
\hline \multirow[b]{2}{*}{ Species } & \multicolumn{23}{|c|}{ Sample number } \\
\hline & $\frac{\stackrel{2}{2}}{\stackrel{\infty}{a}}$ & 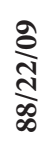 & $\underset{\infty}{\stackrel{a}{d}}$ & 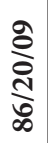 & $\frac{2}{2}$ & 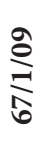 & $\underset{\infty}{\stackrel{\infty}{\infty}}$ & $\frac{\substack{d \\
\infty}}{0}$ & $\frac{g}{\frac{g}{a}}$ & $\underset{\infty}{\stackrel{2}{\curvearrowright}}$ & $\underset{\substack{2 \\
ٍ}}{\stackrel{2}{\delta}}$ & $\frac{\stackrel{\partial}{+}}{\frac{1}{R}}$ & $\underset{\infty}{\stackrel{2}{2}}$ & $\frac{2}{n}$ & 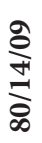 & $\frac{\substack{0 \\
\text { ก }}}{2}$ & $\frac{2}{\stackrel{2}{r}}$ & $\frac{\stackrel{g}{\rho}}{\stackrel{2}{a}}$ & 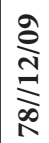 & 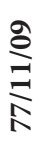 & $\frac{2}{2}$ & $\frac{\frac{a}{a}}{n}$ & $\frac{\substack{\infty \\
+}}{1}$ \\
\hline 1 & 2 & 3 & 4 & 5 & 6 & 7 & 8 & 9 & 10 & 11 & 12 & 13 & 14 & 15 & 16 & 17 & 18 & 19 & 20 & 21 & 22 & 23 & 24 \\
\hline $\begin{array}{l}\text { Trochammina ex. } \\
\text { gr. globigeriniformis } \\
\text { (Jones et Parker) }\end{array}$ & & & & II & & $\mathrm{X}$ & & & I & & & & I & II & $\mathrm{I}$ & II & & & & & & $\mathrm{I}$ & \\
\hline $\begin{array}{l}\text { Trochammina } \\
\text { umiatensis Tappan }\end{array}$ & & & & & $\mathrm{V}$ & & W & $\mathrm{W}$ & & & & $\mathrm{X}$ & I & I & & & & & & & & $\mathrm{X}$ & $\mathrm{V}$ \\
\hline Trochammina sp. & & $\mathrm{V}$ & & & & & $\mathrm{X}$ & I & & & & & II & II & & $\mathrm{H}$ & & & & & I & & I \\
\hline $\begin{array}{l}\text { benthonic calareous } \\
\text { foraminifera (spp.) }\end{array}$ & & & & & & & & & & & & & & & & & & & & & & & I \\
\hline $\begin{array}{l}\text { planctonic } \\
\text { foraminifera (spp.) }\end{array}$ & & & & & & & & & & I & & & & & & & & & & & & & $\mathrm{V}$ \\
\hline
\end{tabular}

The Green shales are overlain by the Globigerina marls, which are characterised by occurrences of harder, massive marls and/or pelitic marly limestones grey in colour on fresh surfaces, but weathering light grey. They form flat lenticular bodies within the clayey and/or marly greenish shales, ranging from few to over a dozen centimetres in thickness and reaching several tens of metres in lateral extent. The sequence containing the marly shales reaches a thickness of circa 3-4 m.

Where the lithotype of hard marl is absent, the transition of the Green shales into the Globigerina marls is gradational and the boundary between these units is arbitrary. Leszczyński (1996) placed the boundary between the Green shales and the Globigerina marls on the first marly mudstone bed, which at the Znamirowice locality (W bank of Lake Rożnów) occurs $6 \mathrm{~m}$ below the base of the Menilite beds. The mudstone-marl strata come into direct contact with the Menilite beds. This contact is sharp and well defined in the section by the appearance of characteristic dark-brown, slightly silicified shales. Mediumto thick-bedded Menilite sandstones appear and begin to accompany the dark shales over a short distance of the section.

\section{FORAMINIFERAL ASSEMBLAGES}

The assemblages consist of taxonomically diversified agglutinated foraminifera, calcareous taxa constitute marginal components of some assemblages (Tab. 1). The cosmopolitan and long-ranging taxa characteristic for the Cretaceous-Eocene interval, like Paratrochamminoides div. sp. (mainly P. heteromorphus (Grzybowski), P. olszewskii (Grzybowski), Trochamminoides div. (mainly T grzybowskii Kaminski et Geroch, T. subcoronatus (Grzybowski), Bathysiphon sp., Nothia sp., Recurvoides div. sp., Cribrostomoides subglobosus (Cushman), Reophax div. sp. (mainly: R. duplex Grzybowski, R. piliulifer Brady), Glomospira div. sp., e.g. G. charoides (Jones et Parker), G. gordialis (Jones et Parker), G. glomerata (Grzybowski), Haplophragmoides div. sp. (mainly $H$. nauticus Kender, Kaminski et Jones, a species first described from West Africa by Kender et al. 2006, and H. walteri (Grzybowski), Ammodiscus div. sp. (mainly: A. cretaceus Reuss, A. peruvianus Berry, A. tenuissimus Grzybowski), Dolgenia sp., Spiroplectammina spectabilis (Grzybowski), Placentammina placenta (Grzybowski), Psammosiphonella div. sp. (mainly P. discreta (Brady), P. cylindrica (Glaessner) dominate in assemblages. 
The taxonomic variability of assemblages from the studied section is low, a feature that is characteristic for deep-water assemblages of Eocene age.

\section{Biostratigraphy}

Foraminifera taken from the Hieroglyphic beds and Green shales section are characteristic for the Middle-Late Eocene interval (Tab. 1). The foraminiferal assemblages were recognized as typical for the biozones: Reticulophragmium amplectens, Ammodiscus latus and Reticulopohragmium rotundidorsatum (biozones on the base of the standard agglutinated foraminiferal biozonations for the Outer Carpathians proposed by Morgiel \& Olszewska 1981, Geroch \& Nowak 1984, and Olszewska 1997). In the studied assemblages, the index taxa have particular biostratigraphical significance.These assemblages contain typical species that are restricted to the Eocene, e.g. $\mathrm{Ha}$ plophragmoides parvulus Blaicher, Haplophragmoides porrectus Malakova, Pseudonodosinella elongata (Grzybowski), and Praesphaerammina subgaleata Vašíček.

Reticulophragmium amplectens zone - Lutetian (Middle Eocene) - the oldest determinated zone, interval between the last occurrence of Saccamminoides carpathicus Geroch, and the first occurrence of Ammodiscus latus Grzybowski. The presence of relatively numerous index taxa is characteristic for this interval.

Assemblages typical for this zone were recovered from the Hieroglyphic beds (samples no. $86 / 20 / 09,87 / 21 / 09$ ) and the Green shales, their lower part of the gray-green muddy sequence with heavy grey mudstone intercalations and the middle part of section, represented by grey-green noncalcareous mudstone (samples no. 67/1/09, 84/18/09, 68/2/09) (Fig. 4).

Reticulophragmium amplectens (Grzybowski) occurs as a common component, and it comprises up to $10 \%$ of the foraminifera in the assemblage. $\mathrm{Ha}$ plophragmoides nauticus Kender, Kaminski et Jones is also numerous.This species, next to Haplophragmoides walteri (Grzybowski) is a common component of Middle Eocene Carpathian foraminiferal assemblages (Waśkowska 2008, Golonka \& Waśkowska 2012). Praesphaerammina subgaleata Vašíček and Popovia beckmanni (Kaminski et Geroch) (represented only by coarse-grained forms) are present. Adercotryma agterbergi Gradstein et Kaminski and Buzasina pacifica (Krasheninnikov) (as a compressed test) also occur. Occasionally Caudammina ovula (Grzybowski) and Hormosina velascoensis (Cushman) are present - these forms are typical for Late Cretaceous and Paleocene, they are very rare in Carpathian Ypressian assemblages in the Saccamminoides carpathicus zone (Olszewska et al. 1996, Waśkowska 2011). Foraminiferal assemblages of the Hieroglyphic beds are slightly different compared to assemblages from the Green shales with respect to the assemblage structure; they exhibit lower taxonomical diversity, and a lower number of specimens. Numerous Spiroplectammina spectabilis (Grzybowski) (up to $15 \%$ of all foraminifera in the assemblage) distinguishes them. A similar proportion of Insculparenula subvesicularis (Hanzlikova) was observed in the sample from the Hieroglyphic beds. The occurrence of Insculptarenula subvesicularis (Hanzlikova) as a significant component (about 7\% all foraminifera) was determined within the Middle Eocene Hieroglyphic beds of the Silesian Nappe in the Szczyrzyc area (Bubík \& Waskowska-Oliwa 2005).

Ammodiscus latus zone - Bartonian (Middle Eocene), the interval between the first occurrence of Ammodiscus latus Grzybowski and the first occurrence of Reticulophragmium rotundidorsatum (Hantken) [=Reticulophragmium gerochi Neagu, 2011].

Assemblages typical for this zone were recognized in the middle part of the Green shales section (samples no 69/3/09, 83/17/09, 70/4/09, $81 / 15 / 09,71 / 5 / 09)$, represented by gray and green mudstones, the calcareous rocks as ex situ elements are most numerous in this part of the profile (Fig. 4).

The species Reticulophragmium amplectens (Grzybowski) irregularly occur in the assemblages. Popovia beckmanni (Kaminski et Geroch) was recognized in assemblages with Ammodiscus latus Grzybowski, its known stratigraphic range reaches up to the Middle Eocene (Kaminski \& Geroch 1987, Olszewska et al. 1996, Kaminski \& Gradstein 2005). Eggerelloides sp. and Bulbobaculites sp. occur as accessory taxa, and Pseudonodosinella elongata (Grzybowski), Haplophragmoides nauticus Kender, Kaminski et Jones, Trochammina umiatensis Tappan and Praesphaerammina subgaleata 
Vašíček are common. Buzasina pacifica (Krasheninnikov) is also present.

Reticulophragmium rotundidorstaium zone - Priabonian (Upper Eocene), interval between the first occurrence of Reticulophragmium rotundidorsatum (Hantken) [= Reticulophragmium gerochi Neagu, 2011] and the first occurrence of Globigerina ampliapertura (Bolli).

Assemblages typical for this zone were found in the middle and upper part of the Green shales section (samples no. 80/14/09, 72/6/09, 73/7/09, $79 / 13 / 09,78 / 12 / 09,77 / 11 / 09,76 / 10 / 09,75 / 9 / 09$, $74 / 8 / 09)$, which was developed as gray-green mudstone/claystones (Fig. 4).

The index taxon - Reticulophragmium rotundidorsatum (Hantken) belongs to the cosmopolitan foraminifera, but in the studied section is represented in low numbers and it is present in assemblages with high taxonomical diversity connected with favorable ecological conditions. It was found in samples no. 80/14/09, 72/6/09, 79/13/09, 76/10/09, other samples were included within the Reticulophragmium rotundidorsatum zone because of their position in the continuous profile. Ammodiscus latus Grzybowski is a common taxon, and it is partly represented by juvenile forms. When the index taxon for the biostratigraphical zone was not present, assemblages could only be assigned to the oldest zone, which may be unreliable for determining the age of samples collected randomly, rather than from a sequence of samples from a continuous profile. Eratidus gerochi Kaminski et Gradstein also occurs in the assemblages.

The calcareous foraminifera were present in samples of green soft marly mudstones/claystones taken from the uppermost part of the Green shales profile. Benthonic forms are represented by poorly preserved Cibicides/Eponides forms with Gyroidinoides globosus (Hagenow), and planktonic forms, among them Subbotina linaperta Finlay are recognized. Arenobulimina dorbignyi (Reuss), which is an agglutinated taxon with calcareous cement, is present in this sample as relatively numerous components.

\section{Foraminiferal assemblages - remarks (Figs 5-8)}

Specific associations. Fragments of tabular tests belonging to the genera Bathysiphon and Nothia are a constant component of the Green shale samples. Specimens of Bathysiphon constitute a few percent to over $50 \%$ of the foraminiferal tests in each sample. Bathysiphon and Nothia are creeping forms existing as epifauna or shallow infauna, and are incorrectly assigned to the morphogroup of erect epifauna (Gooday et al. 1992, Kaminski \& Geroch 1992, Geslin et al. 2004). In the analysed material, the samples obtained from the lowest part of the Green shales succession preserve fragments of large shells of Bathysiphon that are over $2 \mathrm{~mm}$ across. These are the largest foraminifera found in the analysed samples. Slightly smaller specimens were noted by Malecki (1973) within the Ciężkowice beds outcropping in the banks of Rożnów Lake in the Gródek region.

In the lower and middle part of the Green shales succession, Ammosphaeroidina pseudopauciloculata (Mjatliuk) is a common taxon, which constitutes an accessory or main component of the foraminiferal assemblage. Its proportion usually ranges from a few percent to over $10 \%$, but locally may reach $60 \%$, thus forming monospecific assemblages. Other taxa, which are common in the lower and middle part of the Green shales section but disappear in the upper part, include: Pseudonodosinella elongata (Grzybowski), Spiroplectammina spectabilis (Grzybowski), Paraesphaerammina subgaleata Vašíček, and Haplophragmoides parvulus Blaicher. In general, in the uppermost part of the Green shales unit within the Reticulophragmium rotundidorsatum zone, the diversity of the foraminiferal assemblages decreases and the group that increases in quantity is Psammosiphonella, assigned to the morphogroup of erect epifauna.

High-latitude taxa. The foraminiferal assemblages are rather homogeneous taxonomically thanks to the predominance of cosmopolitan forms. The accessory forms found among them are Adercotryma agterbergi Gradstein et Kaminski, Buzasina pacifica (Krasheninnikov), Eratidus gerochi Kaminski et Gradstein, Ammomarginulina aubertae Gradstein et Kaminski, Insculptarenula subvesicularis (Hanzlikova). These taxa are common in, or typical for, high-latitude settings described from the North Sea, North Atlantic, Labrador Sea, Canadian Arctic, and Barents Sea (Gradstein \& Kaminski 1989, 1997, Kaminski \& Gradstein 2005, Setoyama et al. 2011 and citations therein). They occur rarely 
in the Carpathian assemblages of the Silesian and Subsilesian nappes as well as in the autochthonous
Palaeogene succession of Moravia (Bubík 2005b, 2009, Bubík \& Waskowska-Oliwa 2005).

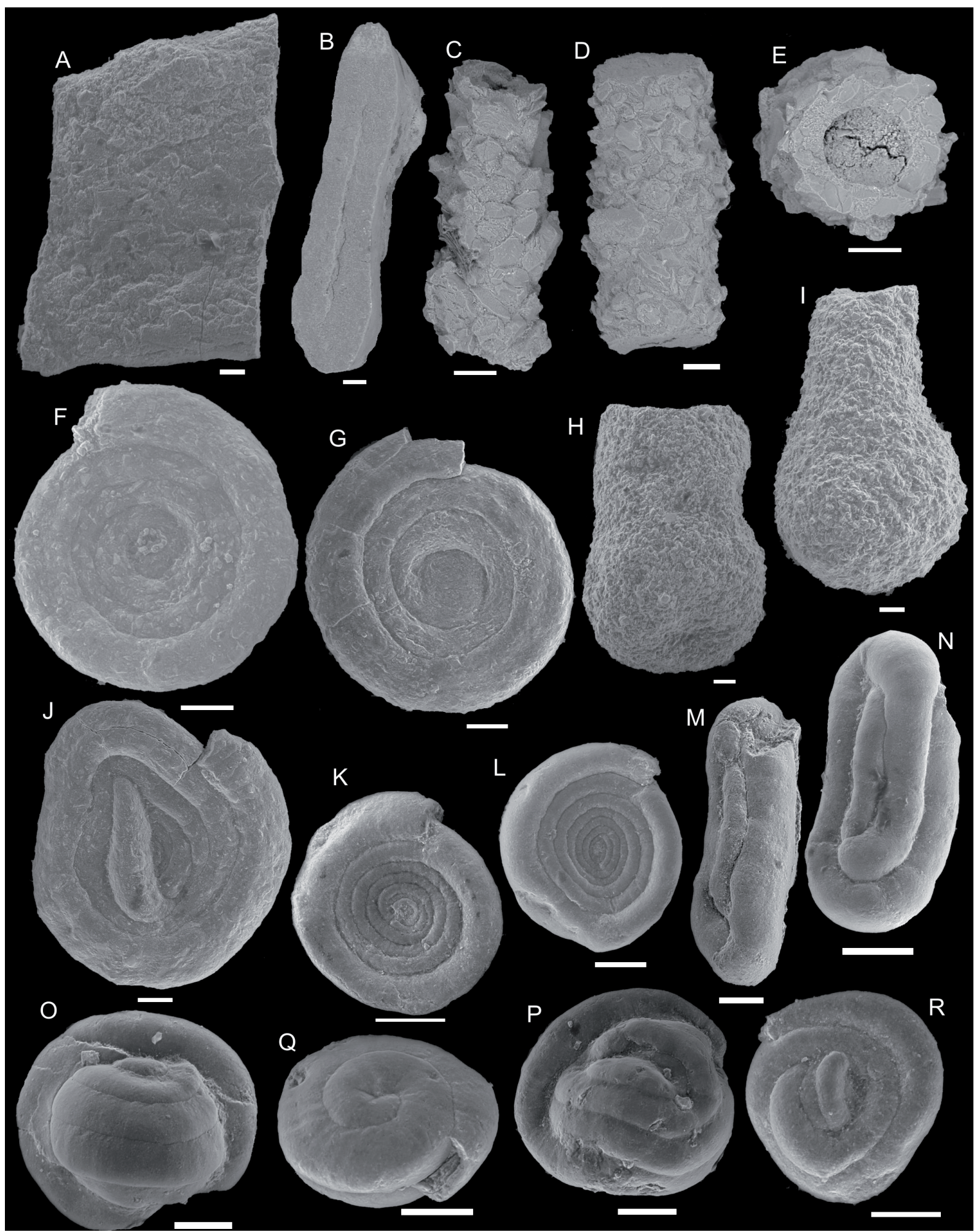

Fig. 5. SEM images of foraminifera from the studied section: A), B) Bathysiphon sp.; C), D), E) Psammosiphonella cylindrica (Glaessner); F), G), J) Ammodiscus (Dolgenia) latus Grzybowski; H), I) Hyperammina sp.; K), L) Ammodiscus cretaceus (Reuss); M), N) Glomospira serpens (Grzybowski); O), P), Q) Glomospira charoides (Jones et Parker); R) Glomospira gordialis (Jones et Parker). Scale bar $=100 \mu \mathrm{m}$ 


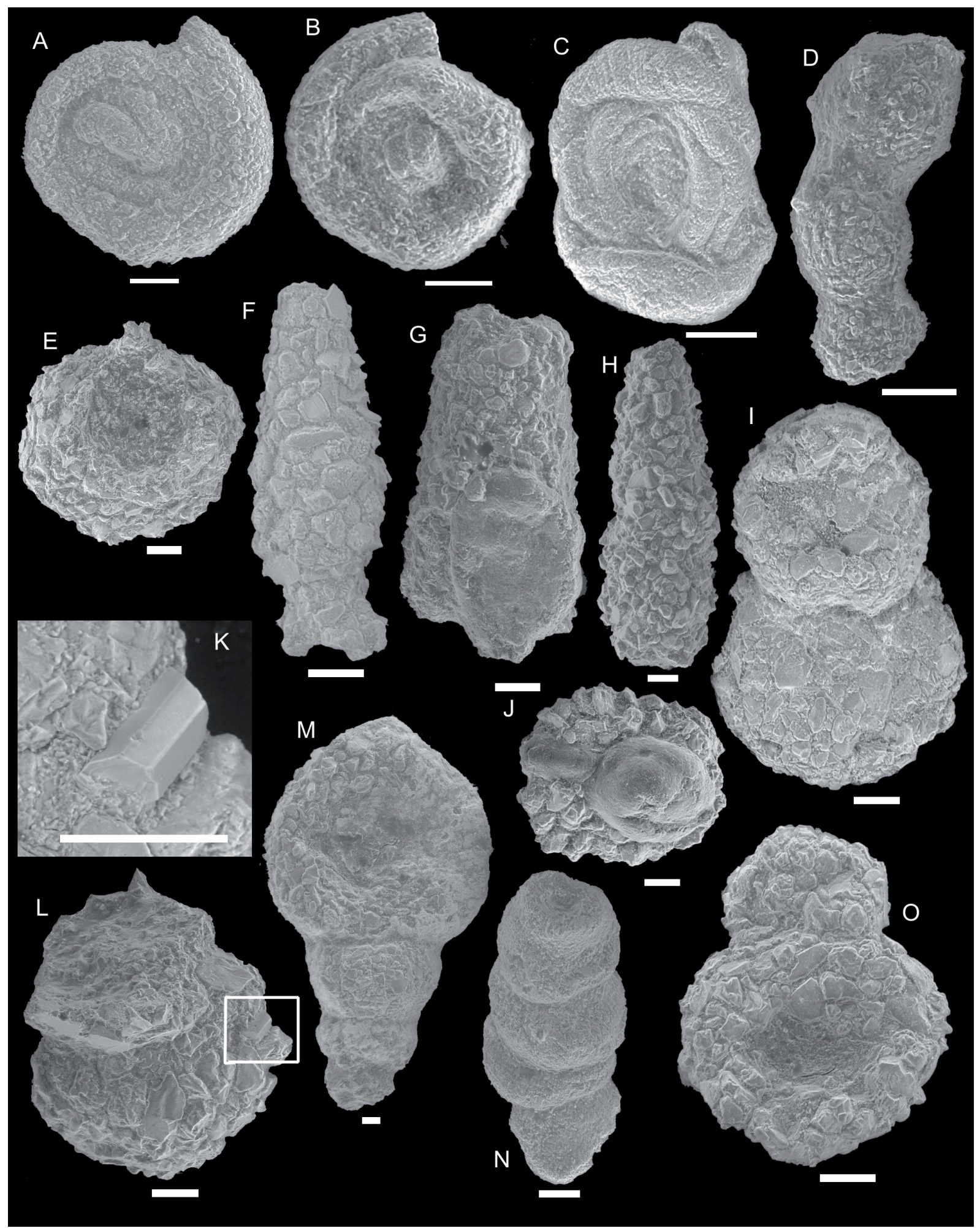

Fig. 6. SEM images of foraminifera from the studied section: A), B), C) Dolgenia sp.; D) Hormosinelloides guttifer (Brady); E) Placentammina placenta (Grzybowski); F), H) Pseudonodosinella elongata (Grzybowski); G) Pseudonodosinella elongata (Grzybowski) with Ammolagena clavata (Jones et Parker); I) Hormosina trinitatensis Cushman et Renz; L) Reophax duplex Grzybowski; J) Ammolagena clavata (Jones et Parker) on single chamber of Reophax; K) tourmaline crystal incorporated within Reophax duplex Grzybowski test; M) Reophax pilulifer Brady; N) Subrepohax pseudoscalaris (Grzybowski); O) Reophax duplex Grzybowski. Scale bar $=100 \mu \mathrm{m}$ 


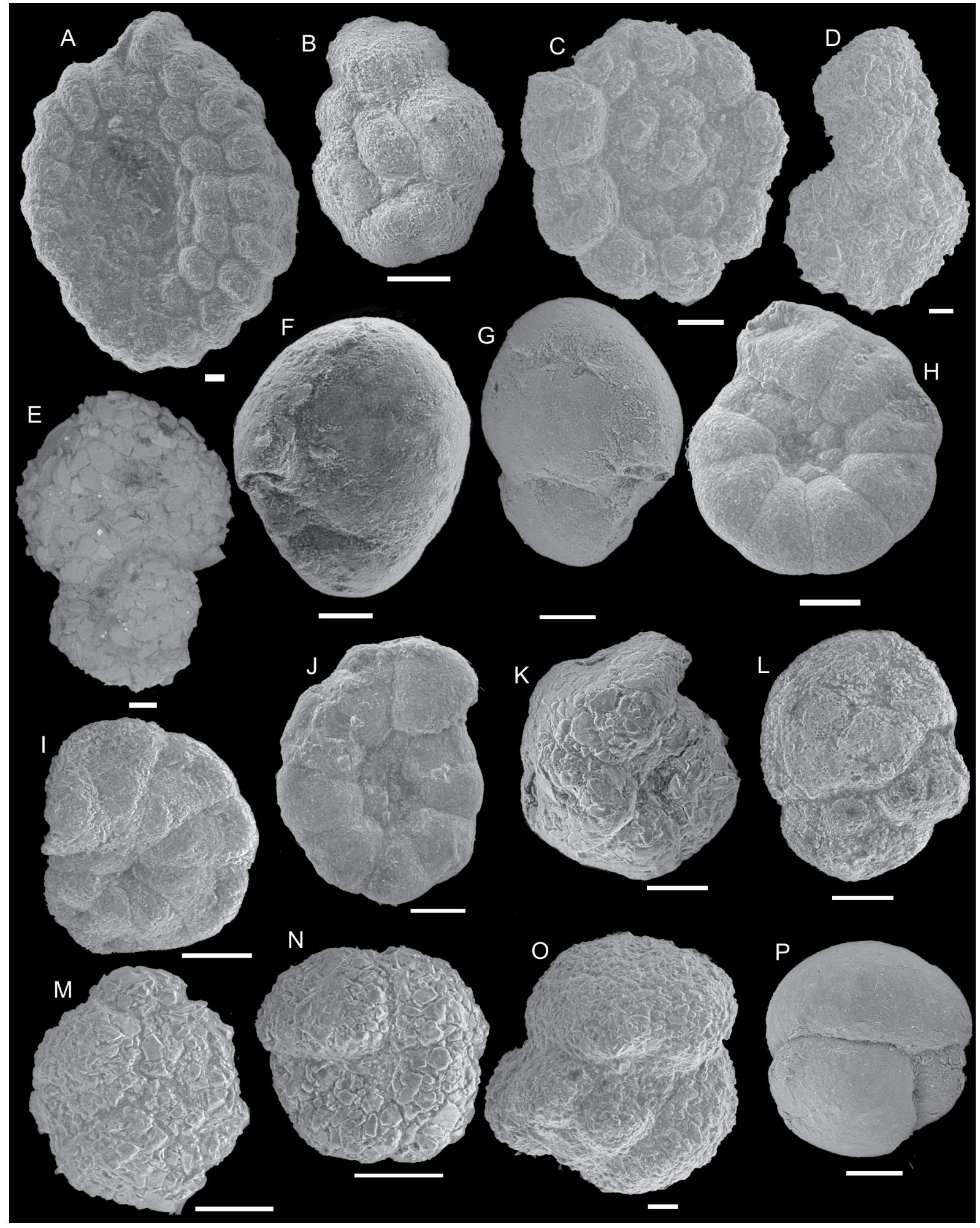

Fig. 7. SEM images of foraminifera from the studied section: A) Trochamminoides elegans (Grzybowski); B) Trochamminoides subcoronatus (Grzybowski); C) Trochamminoides proteus (Karrer); D) Ammobaculites agglutinans d'Orbigny; E) Bulbobaculites sp.; F), G) Buzasina pacifica (Krasheninnikov); H), I), J) Haplophragmoides nauticus Kender, Kaminski et Jones; K) Haplo-
phragmoides sp.; L), P) Ammosphaeroidina pseudopauciloculata (Mjatliuk); M), N) Insculptarenula subvesicularis (Hanzlikova); O) Trochammina umiatensis Tappan. Scale bar $=100 \mu \mathrm{m}$

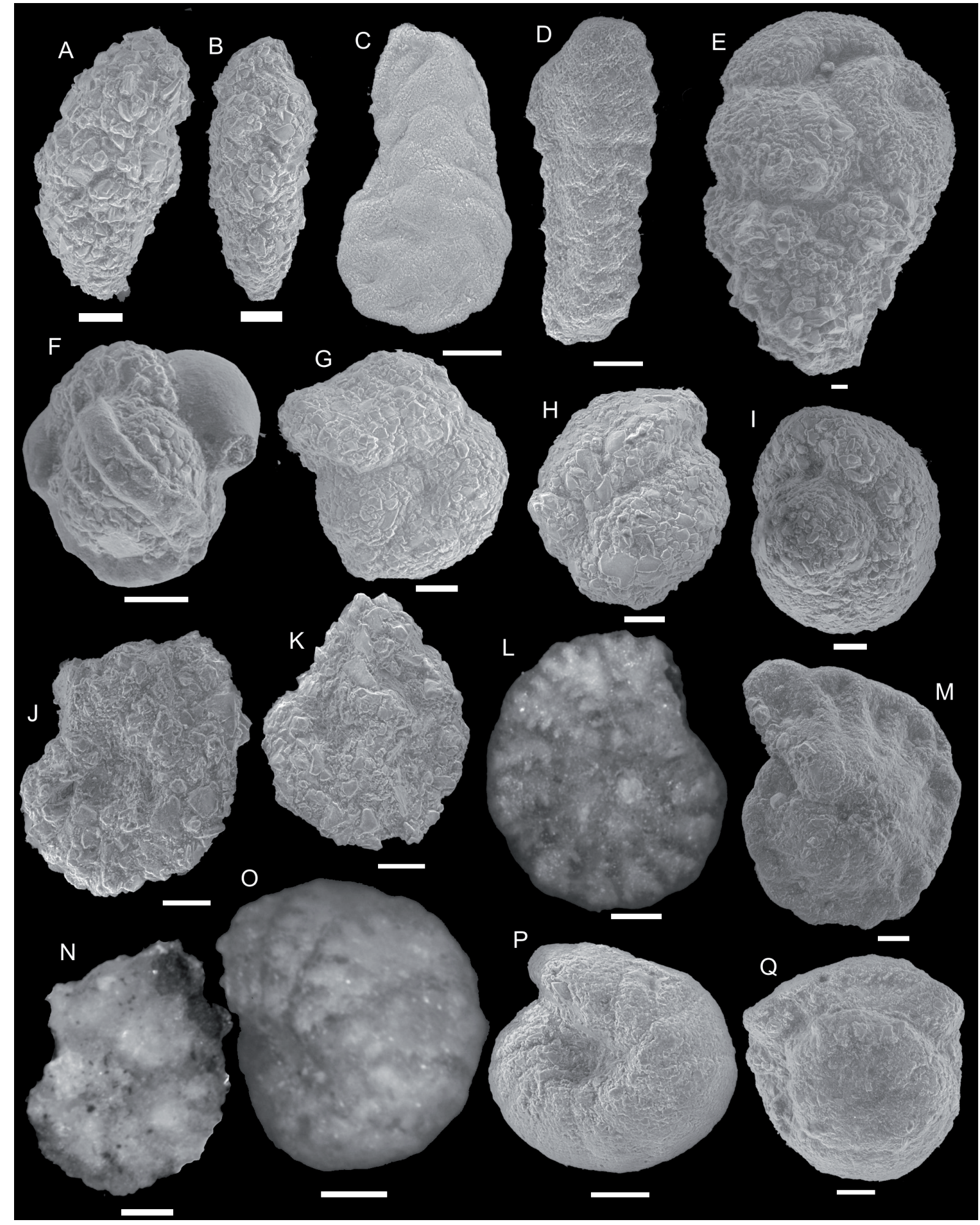

Fig. 8. SEM images of foraminifera from the studied section: A), B) Karrerulina coniformis (Grzybowski); C), D) Spiroplectammina spectabilis (Grzybowski); E) Eggerelloides sp.; F) Ammolagena clavata (Jones et Parker) settled on Recurvoides sp.; G) Recurvoides anormis Mjatliuk; H) Recurvoides sp.; I) Recurvoides nucleolus (Grzybowski); J), K), N) Popovia beckmanni (Kaminski
et Geroch); L), M) Reticulophragmium amplectens (Grzybowski); O), P), Q) Reticulophragmium rotundidorsatum (Hantken). Scale bar $=100 \mu \mathrm{m}$ 
An accessory taxon that occurs in the analysed succession is Buzasina pacifica (Krasheninnikov) characteristic of abyssal and bathyal depth ranges. It is known from the Cretaceous strata (Krasheninnikov 1974, Geroch \& Nowak 1984), but was also observed in the units of Palaeocene (Olszewska et al. 1996, Kaminski \& Gradstein 2005) and Early Eocene age (Bubík 1995a). Its total stratigraphic range was estimated as Albian-Palaeocene (Early Eocene) (Bubík 1995a, Kaminski \& Gradstein 2005). In the analysed samples it occurs in the Middle Eocene, in assemblages of the Lutetian and Priabonian. Therefore, taking into account the occurrence of this taxon in the Outer Carpathians, the full age range of Buzasina pacifica (Krasheninnikov) should be extended into the Middle and Late Eocene, and is currently estimated as Albian-Bartonian. This form occurs in areas of fine-grained substrate (Kaminski \& Gradstein 2005), and in such environments it also appears in the Eocene strata of the Silesian Basin.

Taxonomical remarks. Ammodiscus latus (Grzybowski) and Reticulophragmium rotundidorsatum (Hantken) being index taxa for Eocene biozones should be transferred within taxonomical classification.

One of the diagnostic features of the genus Ammodiscus is planispiral coiling of the long agglutinated test in tube form (Kaminski \& Gradstein 2005 and references therein). Ammodiscus latus (Grzybowski) is a species that displays semi-planispiral coiling. The initial portion, in umbiculus part, is not planispiral, the coiling is irregular, similar to the coil of Glomospira (Fig. 5). Therefore, Ammodiscus latus (Grzybowski) should be transferred to genus Dolgenia Kemper, 1995. In the studied material there are many more Dolgenia specimens, belonging to another species that has not yet been formally described (Fig. 6). These Dolgenia specimens occur in most of the samples from the studied section and constitute an important component of the benthic foraminiferal assemblage.

Reticulophragmium rotundidorsatum (Hantken) [= Reticulophragmium gerochi Neagu, 2011] is an important biostratigraphical taxon, commonly determined in the Carpathians. Neagu et al. (2011) described the species Reticulophragmium gerochi from the Romanian Eocene strata and pointed out the differences between it and the typical Oligocene Reticulophragmium rotundidorsatum (Hantken) from Hungary. The Carpathian specimens can be referred to Reticulophragmium gerochi Neagu, Popescu, Crihan et Popescu, which now constitutes the new name for the older, smaller, more primitive variety of Reticulophragmium rotundidorsatum (Hantken). In this paper, the new nomenclature is not yet used, because the new taxonomical reassignment of Ammodiscus latus (Grzybowski) to Dolgenia lata (Grzybowski) and the Eocene variety of Reticulophragmium rotundidorsatum (Hantken) to Reticulophragmium gerochi Neagu et al. will resulted in a change of the well-established Eocene biozones names, which are widely recognized in the Carpathians. This problem requires a separate discussion.

Agglutinated tourmalines. The mineralogical composition of coarse-grained tests of agglutinating foraminifera from the Green shales is of interest because, next to the common component of quartz grains, they also contain tourmaline crystals (Waśkowska \& Łodziński 2011a, b). Tourmaline is a common building component of the agglutinating tests observed in the assemblages from the upper part of the Hieroglyphic beds.

\section{HIEROGLYPHIC BEDS VERSUS “GREEN SHALES”}

The Hieroglyphic beds identified within the Silesian Nappe constitute a characteristic lithostratigraphic unit of Eocene age with a thickness estimated to be ca. $200 \mathrm{~m}$. It is represented by a thin-bedded flysch sequence, in which thin sandstone beds are a subordinate, but an important part of the succession (e.g. Świdzińki 1948, Bieda et al. 1963, Geroch et al. 1967, Czaplicka et al. 1968, Leszczyński 1985, Cieszkowski 1992, Leszczyński \& Radomski 1994, Golonka \& Waśkowska-Oliwa 2007). In the upper part of the Hieroglyphic beds, or succeeding immediately above, are the "Green shales" considered as a separate informal lithostratigraphic unit reaching $30 \mathrm{~m}$ in thickness (Bieda et al.1963, Geroch et al. 1967, Leszczyński 1997). The Green shales unit is also identified in the Rożnów Lake and Ciężkowice regions as 
a succession of grey-green shales devoid of sandstone interbeds (Burtan \& Skoczylas-Ciszewska 1963, Koszarski et al. 1964, Kuciński 1965, Koszarski 1966, Koszarski \& Żytko 1966, Cieszkowski et al. 1981). The unit is calcareous in its upper part (Cieszkowski et al. 1991, Cieszkowski 1992, Leszczyński 1996) where it also contains intrabasinal slump-related limestone clasts (Cieszkowski 1992, Leszczyński \& Radomski 1994).

Within the investigated area between Sienna and Gródek, the strata that occur between the Ciężkowice sandstone and the Globigerina marls and/or Menilite beds are represented by a relatively thin package of flysch succession of shales interbedded with sandstones of the Hieroglyphic beds, and a succeeding complex of shales (Figs 2, 4). Their lithological character matches the features known from other regions of the Silesian Nappe, however their thickness and stratigraphic range does not match the lithostratigraphic standard of this tectonic unit. The thickness of the Hieroglyphic beds amounts to $20 \mathrm{~m}$, which is several times lower than in other regions. The thickness of the succeeding Green shale unit reaches circa $150 \mathrm{~m}$ (Figs 2, 4). The biostratigraphic data indicate that in the Rożnów Lake region, the Hieroglyphic beds originated in the Lutetian, and the Green shales - from the Middle to Upper Eocene, in the interval Lutetian-Priabonian (the Reticulophragmium amplectens, Ammodiscus latus, Reticulophragmium rotundidorsatum zones). However, the age documentation of the Silesian Nappe Green shales analysed in other areas gives a narrower age interval: the Upper Eocene (Jurkiewicz 1959, Wdowiarz 1965, Koszarski 1966, Koszarski \& Żytko 1966, Geroch et al. 1967, Cieszkowski et al. 1981, Geroch \& Koszarski 1988, Cieszkowski 1992) locally descends down to the Bartonian (Leszczyński \& Radomski 1994). The reported micropalaeontological data provide foraminiferal inventories that include the index forms, i.e. Ammodiscus latus Grzybowski and Reticulophragmium rotundidorsatum Hantken (Geroch et al. 1967, Cieszkowski 1992). The co-occurrence of these species is characteristic for the Priabonian (Jurkiewicz 1967, Morgiel \& Szymakowska 1978, Morgiel \& Olszewska 1981, Geroch \&Nowak 1984, Olszewska et al. 1996, Olszewska 1997) and is equivalent to the Reticulophragmium rotundidorsatum zone. The upper boundary of the Green shales to the overlying Globigerina marls is isochronous and has a Late Eocene age (e.g. Bieda et al. 1963, Koszarski 1963, Burtan \& Skoczylas-Ciszewska 1966, Geroch et al. 1967, Geroch \& Koszarski 1988, Cieszkowski 1992, Leszczyński \& Radomski 1994, Leszczyński 1996, Olszewska 1997), whereas the lower boundary extends down to the Middle Eocene. These age relations suggest that the deposition of the Green shales in some parts of the Silesian Basin started already in the Middle Eocene, which was related to the local decline in turbidite sedimentation characteristic for the Hieroglyphic beds. However, it should be stressed that the total stratigraphic range (the Lutetian-Priabonian) and the thickness of the Hieroglyphic beds together with the overlying Green shales in what is now the Rożnów Lake region matches the regional standard for the Silesian Nappe. The difference is only reflected by the thickness proportions of these units.

A deficiency in siliciclastic detritus may be related not only to the quietness of the environment but also to the deficiency of sandy material supplied to the selected part of the basin.

In the lithological section of the Silesian Nappe from the Gródek region, the Green shales occur partly in the position of the Hieroglyphic beds, the thickness of which is locally reduced. The lateral facies transition results from lateral variations of the depositional regimes in the deep marine flysch basin. These in turn are controlled by the geometry and tectonics of the shelf and slope, distance to the source area, the quantity and composition of terrigenous material supplied to the basin, the dynamics of sediment gravity flows, the physiography of their distributary system, a.s.o. The strata of the Hieroglyphic beds and the Green shales were deposited during intervals of relative quiescence, at considerable depths corresponding to the CCD. The lithological basis for their identification is the presence or absence of thin-bedded turbidite sandstones, which is controlled by the range of reach of the turbidity currents supplying the arenaceous material into this part of the basin. The predominant background facies of the argillites with which sandstones of the Hieroglyphic beds are associated, are equivalent to 
the deposits defined as the Green shales that occur above. The latter were deposited in low energy conditions, under extremely limited influence of turbidity currents bringing the arenaceous material, or entirely beyond their influence. The sedimentary structures that occur in the lower part of the Green shales interval are limited to parallel lamination at macroscopic and microscopic scale, which reflect the influence of extremely diluted turbidity currents bringing in small amounts of siliciclastic detritus and controlling its distribution within the sedimentary packages. The middle and upper parts of the succession, represented by massive mudstone and claystone with subordinate amounts of randomly distributed detrital components, results from hemipelagic deposition. Depending on conditions within the sedimentary environment and position in the basin, the Green shales are the lateral facies equivalent of the Hieroglyphic beds and express the gradually changing conditions of deposition.

In the Sienna area, thin packages of thin-bedded turbidites reminiscent of the Hieroglyphic beds occur within the Green shales. However, detailed field investigations indicate that they represent large elements/olistoliths of an extensive olistostrome, which at this locality are incorporated into the Green shales. There are olistoliths of red variegated shale (Ślączka 1963, Cieszkowski 1992) as well as packets of Ciężkowice-type deposits, gray- and green mudstones, thin- and medium bedded flysch with calcareous banded sandstones (also of Eocene age), armored balls and crystalline magmatic and metamorphic rocs as clasts within matrix, which have to be derived from the more southern part of the Silesian Basin (Cieszkowski et al. 2010). In several instances, the limited outcrop size does not enable us to observe contacts between the olistoliths and the hosting green shale (Cieszkowski 1992, Cieszkowski \& Waśkowska 2013a, b). This, in the case of casual and locally limited observations, may result in interpretations of these turbidite bodies as interbeds within the green shale complex. There are also lateral changes of the Hieroglyphic beds in the studied area, they contain intercalations of thick-bedded sandstones more than $0.5 \mathrm{~m}$ thick, and on the western bank of the Lake (Znamirowice) directly above Ciężkowice sandstone there are complex of grey and green shales with intercalations of thin-bedded sandstones only (Ślączka1964).

\section{CONCLUSIONS}

In the Rożnów Lake area, the strata overlying the Ciężkowice sandstones and succeeded by the Globigerina marls are developed as a relatively thin complex of thin-bedded flysch with intercalations of medium- and thick-bedded turbidites (the Hieroglyphic beds) and overlying it thick complex of the so-called Green shales.

The age of the Hieroglyphic beds in this area was assessed on the basis of agglutinated foraminiferal assemblages, which represent the Middle Eocene Reticulophragmium amplectens zone (the Lutetian). The overlying Green shales, which were in the literature considered as exclusively Late Eocene in age, were in fact deposited between the Lutetian and the Priabonian (the Middle to Late Eocene) (Reticulophragmium amplectens, Ammodiscus latus, Reticulophragmium rotundidorsatum zones). The upper boundary of the Green shales is isochronous, but the lower boundary appears diachronous, with sedimentation beginning in the Middle Eocene (the Lutetian).

The early deposition of the Green shales, which are age equivalent to the middle and upper Hieroglyphic beds in other parts of the Silesian Nappe, was related to laterally variable conditions of deposition in the Silesian Basin. From the Middle Eocene, the Hieroglyphic beds were laterally replaced by the Green shale facies deposited in low energy conditions, under greatly limited influence (or entirely beyond the influence) of turbidity currents transporting the arenaceous detritus.

In the foraminiferal assemblages, accessory deep marine taxa are present that are known from the Early Palaeogene and are common in highlatitude regions. These species have migrated into the Carpathian Basins.

A new stratigraphic range was established for Buzasina pacifica (Krasheninnikov), which occurs in the interval Albian-Priabonian.

The author is grateful for helpful discussions with Jan Golonka (AGH) and Marek Cieszkowski (UJ). Renata Stadnik (AGH) and Stawomir Bębenek (AGH) participated in field work, and Teresa 
Wójcik (AGH) undertook laboratory work. SEM images were made in the Scanning Microscopy Laboratory WGGiOS AGH, calcium carbonate content was defined in the Trace Element Analysis Lab WGiOS AGH by Jadwiga Cyrana, determination of TOC was made in the Department of Geology and Geochemistry SG. Andrzej Ślaczka (UJ) and Michael A. Kaminski (KFUPM-UCL) reviewed a draft of the manuscript.

This research has been financially supported by AGH University of Science and Technology in Krakow grant no. 11.11.140.173.

\section{REFERENCES}

Bieda F., Geroch S., Koszarski L., Książkiewicz M. \& Żytko K., 1963. Stratigraphie des Karpates externes polonaises. Biuletyn Instytutu Geologicznego, 181, 1-174.

Bogacz K., Dziewański J., Jednorowska A. \& Węcławik S., 1979. Osady paleogenu płaszczowiny magurskiej w rejonie Owczar (Beskid Niski) [Paleogene deposits of the Magura nappe near Owczary (Polish Flysch Carpathians)]. Rocznik Polskiego Towarzystwa Geologicznego, 59, 43-65 [with English abstract].

Bubík M., 1995a. Cretaceous to Paleogene agglutinated foraminifera of the Bílé Karpaty unit (West Carpathians, Czech Republic). [in:] Kaminski M.A., Geroch S. \& Gasiński M.A. (eds), Proceedings of the Fourth International Workshop on Agglutinated Foraminifera, Grzybowski Foundation Special Publication, 3, 71-116.

Bubík M., 1995b. Výskyt rodu Eratidus (Foraminifera) $\mathrm{v}$ eocénu moravského flyše [Occurrence of the genus Eratidus (Foraminifera) in the Eocene of the Moravian flysch]. Geologické výzkumy na Moravě a ve Slezsku, 2, $17-18$.

Bubík M., 2009. Nové a málo známé aglutinované foraminifery z paleogé nu jižní Moravy [New and less-known agglutinated Foraminifera from the Paleogene of southern Moravia]. Zprávy o geologických výzkumech v roce 2008, 2-8, 82-85 [with English abstract].

Bubík M. \& Waskowska-Oliwa A., 2005. Boreal agglutinated foraminifera in the Paleogene of the Outer Flysch Carpathians - preliminary results. [in:] Coccioni R., Kaminski M.A. \& Marsili A. (eds), Proceedings of the Seventh International Workshop on Agglutinated Foraminifera, Urbino Italy - October 2-8, 2005. Abstracts Volume, Grzybowski Foundation Special Publication, 13, 9-11.

Burtan J. \& Skoczylas-Ciszewska K., 1964. Szczegółowa mapa geologiczna Polski, region Karpat i Przedgórza, wydanie tymczasowe, skala 1: 50 000, arkusz Męcina. Wydawnictwa Geologiczne, Warszawa.

Burtan J., Golonka J., Oszczypko N., Paul Z. \& Ślączka A., 1981. Mapa geologiczna Polski, B - Mapa bez utworów czwartorzędowych, w skali 1: 200 000, arkusz Nowy Sacz. Wydawnictwa Geologiczne, Warszawa.

Cieszkowski M., 1992. Strefa Michalczowej - nowa jednostka strefy przedmagurskiej w Zachodnich Karpatach Fliszowych i jej geologiczne otoczenie [Michalczowa zone: a new unit of Fore-Magura zone, Outer Carpathians, South Poland]. Zeszyty Naukowe Akademii Górniczo-Hutniczej im. Stanisława Staszica, 1463, Geologia, 18, 1-125 [with English summary].

Cieszkowski M. \& Waśkowska A., 2013a. Olistostrome deposits in the Eocene Hieroglyphic Formation (Silesian Nappe, Outer Carpathians). [in:] Bąk M., Kowal-Kasprzyk J., Waśkowska A. \& Kaminski M.A. (eds), $14^{\text {th }}$ Czech-Slovak-Polish Paleontological Conference and $9^{\text {th }}$ Micropaleontological Workshop. Abstracts Volume, Grzybowski Foundation Special Publication, 19, 10-11.

Cieszkowski M. \& Waśkowska A., 2013b. Olistostrome deposits in the Eocene Hieroglyphic Formation (Silesian Nappe, Outer Carpathians). [in:] Broska I. \& Tomašových A., Geological evolution of the Western Carpathians: new ideas in the field of inter-regional correlations. Smolenice, Slovak Republic, October 16-19, 2013. Abstracts Book, Geological Institute, Slovak Academy of Sciences, Bratislava, 39-40.

Cieszkowski M., Koszarski A., Leszczyński S., Michalik M., Radomski A. \& Szulc L., 1991. Szczegółowa mapa geologiczna Polski 1:50 000, arkusz Ciężkowice. Państwowy Instytut Geologiczny, Warszawa.

Cieszkowski M., Ślączka A. \& Waśkowska A., 2010. Eocene olistostrome in the Silesian Nappe at the shore of Rożnów Lake, Outer Carpathians, Poland. Mineralia Slovaca-Geovestník, 42, 2, 508.

Czaplicka J., Juskowiak O., Ryka W., Kilian Z., Tomczykowa E., Tomczyk H., Pajchlowa M., Stopa S.Z., Kozłowski S., Kotański Z., Kopik J., Malinowska l., Cieśliński S., Woźny E., Głodek J., Krajewski S., 1968. Słownik stratygraficzny.Wydawnictwa Geologiczne, Warszawa.

Geroch S., 1960. Zespoły mikrofauny z kredy i paleogenu serii śląskiej w Beskidzie Śląskim. Biuletyn Instytutu Geologicznego, 153, 5, 7-73 [with English summary].

Geroch S. \& Koszarski L., 1988. Agglutinated foraminiferal stratigraphy of the Silesian flysch through. Abhandlungen der Geologischen Bundesanstalt, 41, 73-79.

Geroch S. \& Nowak W., 1984. Proposal of zonation for the Late Tithonian-Late Eocene, based upon arenaceous Foraminifera from the Outer Carpathians, Poland. [in:] Oertli H.J. (ed.), BENTHOS '83: $2^{\text {nd }}$ International Symposium on Benthic Foraminifera (Pau, April 11-15, 1983), 225-239.

Geroch S., Jednorowska A., Książkiewicz M. \& Liszkowa J., 1967. Stratigraphy based upon microfauna in the Western Polish Carpathians. Biuletyn Instytutu Geologicznego, 211, 185-282.

Geslin E., Heinz P. \& Hemleben C., 2004. Behaviour of Bathysiphon sp. and Siphonammina bertholdii n. gen n.sp. under controlled oxygen conditions in the laboratory; implication for bioturbation. [in:] Bubík M. \& Kaminski M.A. (eds), Proceedings of the Sixth International Workshop on Agglutinated Foraminifera. Prague, Czech Republic, September 1-7, 2001, Grzybowski Foundation Special Publication, 8, 105-118.

Golonka J. \& Waśkowska A., 2012. The Beloveža Formation of the Raca Unit in the Beskid Niski Mts. (Magura Nappe, Polish Flysch Carpathians) and adjacent parts of Slovakia and their equivalents in the western part of the Magura Nappe; remarks on the Beloveža Formation - Hieroglyphic Beds controversy. Geological Quarterly, $56,4,821-832$. 
Golonka J. \& Waśkowska-Oliwa A., 2007. Stratygrafia polskich Karpat fliszowych pomiędzy Bielskiem-Białą a Nowym Targiem [Stratigraphy of the Polish Flysch Carpathians between Bielsko-Biała and Nowy Targ]. Geologia. Kwartalnik Akademii Górniczo-Hutniczej im. Stanisława Staszica w Krakowie, 33, 5-28 [with English summary].

Golonka J., Ślączka A., Waśkowska A., Krobicki M. \& Cieszkowski M., 2013. Budowa geologiczna zachodniej części polskich Karpat zewnętrznych. [in:] Krobicki M. \& Feldman-Olszewska A. (eds), Głębokomorska sedymentacja fliszowa. Sedymentologiczne aspekty historii basenów karpackich. V Polska Konferencja Sedymentologiczna POKOS 5'2013, 11-62.

Gooday A.J., Levin L.A., Thomas C.L. \& Hecker B., 1992. The distribution and ecology of Bathysiphon filiformis Sars and B. major de Folin (Protista, Foraminiferida) on the continental slope off North Carolina. Journal of Foraminiferal Research, 22, 2, 129-146.

Gradstein F.M. \& Kaminski M.A., 1989.Taxonomy and biostratigraphy of new and emended species of Cenozoic deep-water agglutinated foraminifera from the Labrador and North seas. Micropaleontology, 35, 72-92.

Gradstein F.M. \& Kaminski M.A., 1997. New species of Paleogene deep-water agglutinated foraminifera from the North Sea and Norwegian Sea. Annales Socetatis Geologorum Poloniae, 67, 217-229.

Guzik K. \& Pożaryski W., 1949. Fałd Biecza (Karpaty Środkowe). Biuletyn - Państwowy Instytut Geologiczny, 53, $1-33$

Jurkiewicz H., 1959. Poziomy otwornicowe paleogenu wschodniej części jednostki śląskiej. Rocznik Polskiego Towarzystwa Geologicznego, 29, 235-263.

Jurkiewicz H., 1967. Otwornice paleogenu podmenilitowego polskich Karpat środkowych. Biuletyn Instytutu Geologicznego, 210, 5-128.

Kaminski M.A. \& Geroch S., 1987. Two new species of Phenacophragma from the Paleogene of the Trinidad and Poland. Micropaleontology, 33, 185-188.

Kaminski M.A. \& Geroch S., 1992.The morphology, paleoecology and systematics of Nothia excelsa (Grzybowski), a deep-water agglutinated foraminifer. Annales Societatis Geologorum Poloniae, 62, 255-267.

Kaminski M.A. \& Gradstein F.M., 2005. Atlas of Paleogene cosmopolitan deep-water agglutinated foraminifera. Grzybowski Foundation Special Publication, 10, 1-547.

Kender S., Kaminski M.A. \& Jones R.W. 2006. Four new species of deep-water agglutinated foraminifera from the Oligocene - Miocene of the Congo Fan (offshore Angola). Micropaleontology, 52, 5, 465-470.

Koszarski L., 1966. Szczegółowa mapa geologiczna Polski, region Karpat i Przedgórza, wydanie tymczasowe, skala 1: 50 000, arkusz Rzepiennik. Wydawnictwa Geologiczne, Warszawa.

Koszarski L. \& Żytko K., 1966. Szczegółowa mapa geologiczna Polski, region Karpat i Przedgórza, wydanie tymczasowe, skala 1: 50 000, arkusz Jasło. Wydawnictwa Geologiczne, Warszawa.

Koszarski L., Kuciński T. \& Szymakowska F., 1964. Szczegółowa mapa geologiczna Polski, region Karpat i Przedgórza, wydanie tymczasowe, skala 1: 50 000, arkusz Pilzno. Wydawnictwa Geologiczne, Warszawa.
Krasheninnikov V.A., 1974. Upper Cretaceous benthonic agglutinated foraminifera, Leg 27 of the Deep Sea Drilling Project. Initial Reports of Deep Sea Drilling Project, 27, 637-661.

Książkiewicz M., 1972. Budowa geologiczna Polski. T. 4: Tektonika. Cz. 3: Karpaty. Wydawnictwo Geologiczne, Warszawa.

Kuciński T., 1965. Szczegółowa mapa geologiczna Polski, region Karpat i Przedgórza, wydanie tymczasowe, skala 1: 50 000, arkusz Tuchów. Wydawnictwa Geologiczne, Warszawa.

Leszczyński S., 1985.Piaskowce pasiaste z warstw hieroglifowych w Jastrzębiej k. Ciężkowic [Banded sandstones of the Hieroglyphic Beds at Jastrzębia near Ciężkowice]. Kwartalnik Geologiczny, 29, 2, 395-403 [with English summary].

Leszczyński S., 1996. Origin of lithological variation in the sequence of the Sub-Menilite Globigerina Marl at Znamirowice (Eocene-Oligocene transition, Polish Outer Carpathians). Annales Societatis Geologorum Poloniae, 66, 245-267.

Leszczyński S., 1997. Origin of the Sub-Menilite Globigerina Marl (Eocene-Oligocene transition) in the Polish Outer Carpathians. Annales Societatis Geologorum Poloniae, 67, 367-427.

Leszczyński S. \& Radomski A., 1994. Objaśnienia do Szczegółowej mapy geologicznej Polski w skali 1: 50 000, arkusz Ciężkowice. Wydawnictwa Geologiczne, Warszawa.

Małecki J., 1973. Bathysiphons from the Eocene of the Carpathian Flysch, Poland. Acta Paleontologica Polonica, 18, 163-174.

Morgiel J. \& Olszewska B., 1981. Biostratigraphy of the Polish External Carpathians based on agglutinated foraminifera. Micropaleontology, 27, 1-30.

Morgiel J. \& Szymakowska F., 1978. Stratygrafia paleocenu i eocenu jednostki skolskiej. Biuletyn Instytutu Geologicznego, 310, 39-171 [with English summary].

Neagu T., Popescu D.P., Crihan L.M. \& Popescu G., 2011. Upper Eocene agglutinated foraminifera from Buciumeni section (Ialomita Valley, Dambovita District, Romania). [in:] Kaminski M.A. \& Filipescu S. (eds), Proceeedings of the Eighth International Workshop on Agglutinated Foraminifera, Grzybowski Foundation Special Publication, $16,151-172$.

Olszewska B., 1997. Foraminiferal biostratigraphy of the Polish Outer Carpathians: A record of basin geohistory. Annales Societatis Geologorum Poloniae, 67, 325-337.

Olszewska B., Odrzywolska-Bieńkowa E., Giel M. D., Pożaryska K. \& Szczechura K., 1996. Eichwald, 1830. [in:] Malinowska L. \& Piwocki M. (red.), Atlas skamieniałości przewodnich i charakterystycznych. Cz. 3a: Kenozoik, trzeciorzęd, paleogen, serie: Budowa geologiczna Polski, t. 3, Polska Agencja Ekologiczna, Warszawa, 45-215.

Paul K. \& Tietze E., 1879. Neue Studien in der Sandsteinzone der Karpathen. Jahrbuch der Kaiserlich-königlichen geologischen Reichsanstalt, 29, 189-304.

Ryłko W., 2004. Karpaty. [in:] Peryt T. \& Piwocki M. (red.), Stratygrafia. Cz. 3a: Kenozoik, paleogen, neogen, serie:Budowa geologiczna Polski, t. 1, Państwowy Instytut Geologiczny, Warszawa, 271-321. 
Setoyama E., Kaminski M.A. \& Tyszka J. 2011. Late Cretaceous Agglutinated Foraminifera and Implications for the Biostratigraphy and Palaeoenvironments of the southwestern Barents Sea. [in:] Kaminski M.A. \& Filipescu S. (eds), Proceedings of the Eighth International Workshop on Agglutinated Foraminifera, Grzybowski Foundation Special Publications, 16, 251-309.

Sokołowski S., 1935. Geologia doliny Dunajca pomiędzy Tropiem a Kurowem. Kosmos A, 60, 49-93.

Ślączka A., 1959. Stratygrafia serii śląskiej łuski Bystrego na południe od Baligrodu. Biuletyn Instytutu Geologicznego, 131, 203-286 [with English summary].

Ślączka A., 1963. Pstre łupki z Miłkowej i budowa obszaru otaczającego. Kwartalnik Geologiczny, 7, 295-304 [with English summary].

Ślączka A., 1964. Jednostka śląska między Bilskiem a Tabaszową [Geological structure of the Silesian Unit between Bilsko and Tabaszowa]. Kwartalnik Geologicz$n y, 8,199-210$ [with English summary].

Świdziński H., 1948. Słownik stratygraficzny północnych Karpat fliszowych. Biuletyn Państwowego Instytutu Geologicznego, 37, 1-124.

Waśkowska A., 2011. The Early Eocene Saccamminoides carpathicus Assemblage in the Outer Flysch Carpathians. [in:] Kaminski M.A. \& Filipescu S. (eds), Proceeedings of the Eighth International Workshop on Agglutinated Foraminifera, Grzybowski Foundation Special Publication, 16, 331-341.
Waśkowska-Oliwa A., 2008. Haplophragmoides nauticus Kender, Kaminski \& Jones in the Eocene of the Flysch Carpathians. [in:]: Filipescu S. \& Kaminski M.A. (eds), Eighth International Workshop on Agglutinated Foraminifera, Cluj-Napoca, Romania September 7-13, 2008. Abstract Volume, Grzybowski Foundation Special Publication, 14, 63-64.

Waśkowska A. \& Łodziński M., 2011a. Tourmalines in the tests of deep water agglutinated Reophax pilulifer Brady - preliminary results. [in:] Bąk M., Kaminski M.A. \& Waśkowska A. (eds), Integrating Microfossil Records from the Oceans and Epicontinental Seas, Grzybowski Foundation Special Publication, 17, 138-139.

Waśkowska A. \& Łodziński M., 2011b. Turmaliny jako komponent ścian skorupek głębokowodnych otwornic aglutynujących gatunku Reophax piluluifer Brady - badania wstępne na bazie eoceńskiego materiału z Karpat Zewnętrznych. [in:] Boorova D. (red.), 12. česko-slovensko-pol'ská paleontologická konferencja. Bratislava 20-21 októbra 2011. Zborník príspevkov, 105-106.

Wdowiarz S., 1965. Szczegółowa mapa geologiczna Polski, region Karpat i Przedgórza, wydanie tymczasowe, skala 1: 50 000, arkusz Rymanów. Wydawnictwa Geologiczne, Warszawa.

Węcławik S., 1969. Budowa geologiczna płaszczowiny magurskiej między Uściem Gorlickim a Tyliczem [The geological structure of the Magura Nappe between Uście Gorlickie and Tylicz]. Prace Geologiczne Komisji Nauk Geologicznej PAN, Oddziat w Krakowie, 59, 1-101 [with English summary]. 